\title{
Exploring the Flexibility of MIL-47(V)-Type Materials Using Force Field Molecular Dynamics Simulations
}

\author{
J. Wieme, L. Vanduyfhuys, S. M. J. Rogge, M. Waroquier, and V. Van Speybroeck* \\ Center for Molecular Modeling (CMM), Ghent University, Technologiepark 903, 9052 Zwijnaarde, Belgium
}

Supporting Information

ABSTRACT: The flexibility of three MIL-47(V)-type materials (MIL-47, COMOC-2, and COMOC-3) has been explored by constructing the pressure versus volume and free energy versus volume profiles at various temperatures ranging from 100 to $400 \mathrm{~K}$. This is done with first-principles-based force fields using the recently proposed QuickFF parametrization protocol. Specific terms were added for the materials at hand to describe the asymmetry of the one-dimensional vanadiumoxide chain and to account for the flexibility of the organic linkers. The force fields are used in a series of molecular dynamics simulations at fixed volumes but varying unit cell shapes. The three materials show a distinct pressure-volume behavior, which underlines the ability to tune the mechanical properties by varying the linkers toward different applications such as nanosprings, dampers, and shock absorbers.

\section{INTRODUCTION}

Metal-organic frameworks (MOFs) are a class of nanoporous, crystalline materials consisting of inorganic moieties connected through organic linkers. ${ }^{1-3}$ Their diversity in chemical and physical properties, combined with the possibility to rationally design and synthesize them, makes MOFs an attractive field of research with many potential applications. ${ }^{4-7}$ An intriguing subclass of MOFs are those that display a large structural flexibility induced by various stimuli (temperature, pressure, guests, etc.), the so-called flexible or stimuli-responsive MOFs. ${ }^{7-13}$

Because an increasing number of MOFs are synthesized for the first time each year, ${ }^{14}$ and an even larger number of them have also been hypothesized, ${ }^{15}$ the true challenge consists of identifying materials for specific applications. In this respect, molecular simulations can play a very important role ${ }^{16-19}$ in characterizing materials, understanding certain phenonema, and guiding the development of diverse applications. Although accurate first-principles calculations on MOFs are becoming more and more feasible, ${ }^{18,20}$ in many cases one is interested in time and length scales only accessible with molecular mechanics, i.e., force fields, and not with computationally more expensive electronic structure methods such as density functional theory (DFT). Nonetheless, the disadvantage of using force fields is that they need to be derived and parametrized properly, which is a nontrivial task that is typically accomplished using experimental and/or theoretical input. If the target is the study of stimuli-responsive MOFs, one has to construct a flexible force field, allowing the framework atoms to move, to describe the envisaged structural transformation. To date, several procedures for the construction of flexible force fields for MOFs have been proposed in the literature. ${ }^{21-24}$ However, as recently pointed out by Coudert and Fuchs in a comprehensive review, ${ }^{19}$ there is a need for the systematic development of accurate force fields for stimuliresponsive MOFs. Despite various successful force field studies of stimuli-responsive MOFs (see e.g. refs 25-33), an easy way to obtain these force fields did not exist until recently. A variety of studies is available where generic force fields such as UFF ${ }^{34}$

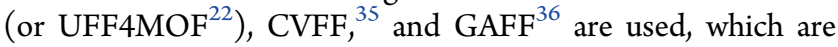
slightly modified for the given purpose. They all succeed in reproducing qualitatively well the expected behavior, or at least give insight in the mechanism, but some features that are inherent to the material require more specific nongeneric force fields. A procedure, hereafter called QuickFF, to generate these force fields was only recently proposed by some of the presenting authors. ${ }^{24}$ In this work, we apply and extend QuickFF and show that it is possible to study the flexibility of guest-free MIL-47-type materials.

The metal-organic framework MIL-47( $\left.\mathrm{V}^{\mathrm{IV}}\right)$ (MIL, materials of Institut Lavoisier) was synthesized for the first time in 2002 by Barthelet et al. ${ }^{37}$ of the Férey group and was originally considered to be rigid under mild working conditions. The structure exhibits a framework pattern similar to that of the MIL-53 series, consisting of one-dimensional (1D) diamondshaped straight channels with a free diameter of about $8 \AA$ and forming infinite chains of corner-sharing $\mathrm{V}^{\mathrm{IV}} \mathrm{O}_{6}$ octahedra connected by 1,4-benzenedicarboxylate (BDC) linkers. The

Received: May 2, 2016

Revised: June 12, 2016

Published: June 13, 2016 

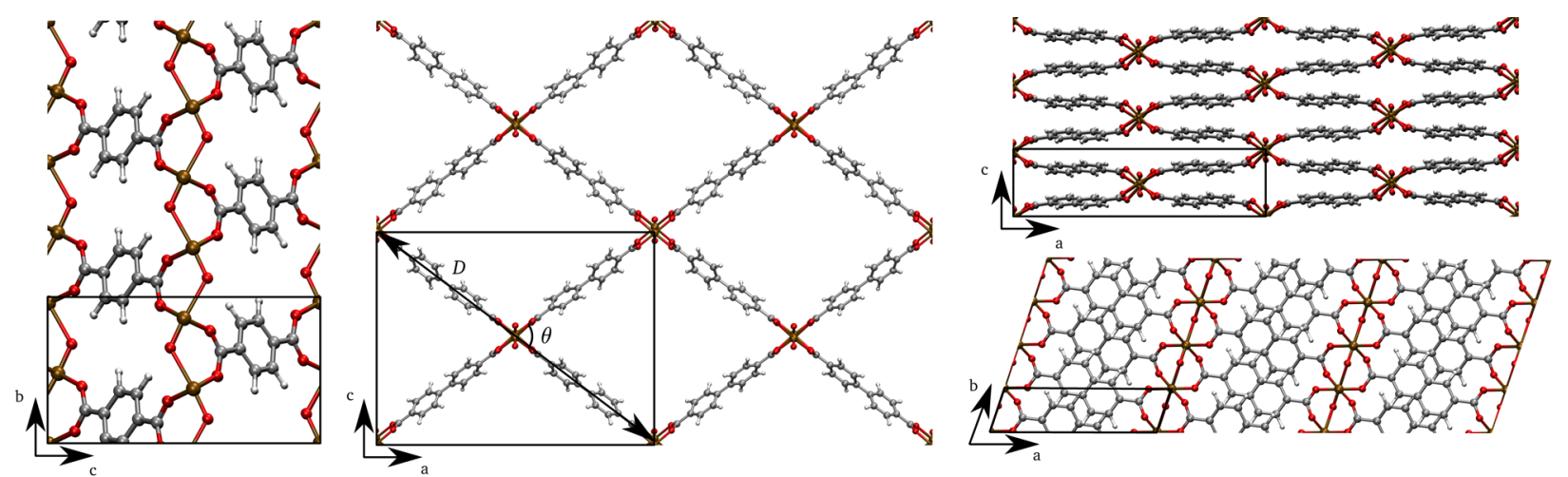

Figure 1. From left to right: MIL-47, COMOC-2, and COMOC-3. The unit cell is indicated, and the interdiagonal distance, $D$, and the pore opening angle, $\theta$, are defined.

most striking difference with the MIL-53 series is the oxidation number of the metal. While in MIL-53 all metals have oxidation number + III, the vanadium metal in MIL-47 has an oxidation number $+\mathrm{IV}$. Direct results are the appearance of an $\mathrm{O}-\mathrm{V}=\mathrm{O}$ vanadium-oxide chain, without hydrogens on the bridging oxygens, and asymmetric $\mathrm{V}-\mathrm{O}$ bond distances. Another difference with the MIL-53 framework is the displaced vanadium chains visible in single-crystal X-ray diffraction. ${ }^{37,38}$

While MIL-47 $\left(\mathrm{V}^{\mathrm{IV}}\right)$ receives the most attention in the literature, other vanadium MOFs have been synthesized and were summarized in a recent review by Van Der Voort et al. ${ }^{39}$ In this work, two variants of MIL- $47\left(\mathrm{~V}^{\mathrm{IV}}\right)$, COMOC- $2\left(\mathrm{~V}^{\mathrm{IV}}\right)^{40}$ and COMOC- $3\left(\mathrm{~V}^{\mathrm{IV}}\right),{ }^{41}$ will also be studied (COMOC, Center for Ordered Materials, Organometallics and Catalysis, Ghent University). These MOFs are the vanadium equivalent of the DUT-5 and DUT $-4{ }^{42}$ topologies (DUT, Dresden University of Technology), which were constructed by Senkovska et al. ${ }^{42}$ with aluminum-oxide chains. Both frameworks have asymmetric vanadium-oxide chains connected with 4,4'-biphenyldicarboxylate (BPDC) linkers for COMOC-2 and 2,6naphtalenedicarboxylate (NDC) linkers for COMOC-3 (see Figure 1). In a synthesis protocol, typically the inorganic moieties are formed in situ, while the organic linkers are preformed, and recent advances in synthesis have made it possible to focus on linker design. ${ }^{43}$ By replacing the linker in MIL-47 with other ditopic carboxylate linkers, a different flexibility behavior can be obtained, which is also observed in our simulations. On the one hand, COMOC-2 is considered to be a bistable MOF because it displays breathing toward certain guest molecules ${ }^{40,44,45}$ in contrast to DUT-5, its rigid $\mathrm{Al}$ analogue. $^{42}$ This peculiar trend is the opposite of the adsorption behavior of MIL-47 as compared to MIL-53(Al), i.e., its flexible $\mathrm{Al}$ analogue. It was also observed that the presence of $\mathrm{V}^{\mathrm{III}}$ compared to a pure COMOC- $2\left(\mathrm{~V}^{\mathrm{IV}}\right)$ increases the rigidity, again in contrast to MIL-47. ${ }^{46}$ Moreover, the COMOC- $2\left(\mathrm{~V}^{\mathrm{IV}}\right)$ sample is a mixture of a large-pore (lp) and narrow-pore (np) phase, ${ }^{40}$ which could indicate that both phases are minima of the free energy surface, similar to the intrinsic bistability of MIL-53(Al) at certain temperatures. ${ }^{47,48}$ This was confirmed with periodic DFT calculations by Liu et al., ${ }^{40}$ and the authors even obtained a third hypothetical, overstretched $n p$ phase. On the other hand, guest-free COMOC-3 has a good chemical and thermal stability and is considered to be nonporous, as observed by $\mathrm{N}_{2}$ sorption measurements. $^{41}$ It remains in the np state upon removal of water molecules. $^{41}$
In general there is only a limited number of studies available on mechanical properties of MOFs. ${ }^{49-52}$ However, the firstprinciples full elastic tensor has been calculated and discussed for MIL- $47^{20,50,53}$ and its $\mathrm{NH}_{2}$ substituted variant, ${ }^{54}$ as well as the influence of the spin configuration on the bulk modulus. ${ }^{55}$ Related to these mechanical properties are some high-end applications in the field of mechanical energy storage. MOFs that undergo structural transitions under pressure are especially interesting. ${ }^{29,56-61}$ One typically distinguishes three different classes of MOFs depending on the amount of dissipated mechanical energy in the compression-decompression cycle: a nanospring (no dissipation, i.e., no hysteresis), a damper (partial dissipation, i.e., reversible transformation with hysteresis), and a shock absorber (total dissipation, i.e., irreversible transformation). In this context, the adjective "reversible" means that the system (i.e., MOF) can be reversed to its initial condition. In the context of thermodynamics, a process exhibiting hysteresis is always irreversible, which in this case is due to the fact that the environment cannot return to its initial condition. Only recently, Yot et al. ${ }^{29}$ proved with mercury porosimetry measurements that MIL-47 is in fact a stimuli-responsive MOF, displaying a structural transformation under a large mechanical pressure. This so-called pressureinduced breathing is associated with a volume reduction of approximately $38 \%$ going from a lp state to a np state. Very similar experimental results were obtained by Rodriguez et al. ${ }^{61}$ with a silicon oil pressure medium. The transition is reversible with hysteresis, making MIL-47 a candidate for damper applications, although more recently A520 or MIL-53(Al)-FA was identified to be a better choice because of a higher amount of energy absorbed per unit of mass. ${ }^{60}$

Different thermodynamic studies have been devoted to the construction of free energy profiles and phase diagrams in terms of guest-host interactions for the MIL series. ${ }^{62-69}$ However, free energy profiles of the guest-free frameworks have only seldomly been studied ${ }^{67,69,70}$ and are completely absent for MIL-47 or MIL-47-type materials. In this work, we also aim to fill this gap, using a recently proposed procedure by Rogge et al., ${ }^{70}$ which was applied to both rigid and flexible MOFs. This method consists of extracting the averaged pressure at each volume by performing independent molecular dynamics (MD) simulations at different volume points on a grid in a new ensemble, the $\left(N, V, \boldsymbol{\sigma}_{\mathbf{a}}=\mathbf{0}, T\right)$ ensemble. The free energy profile as a function of the volume can then be obtained via thermodynamic integration. ${ }^{71}$ 

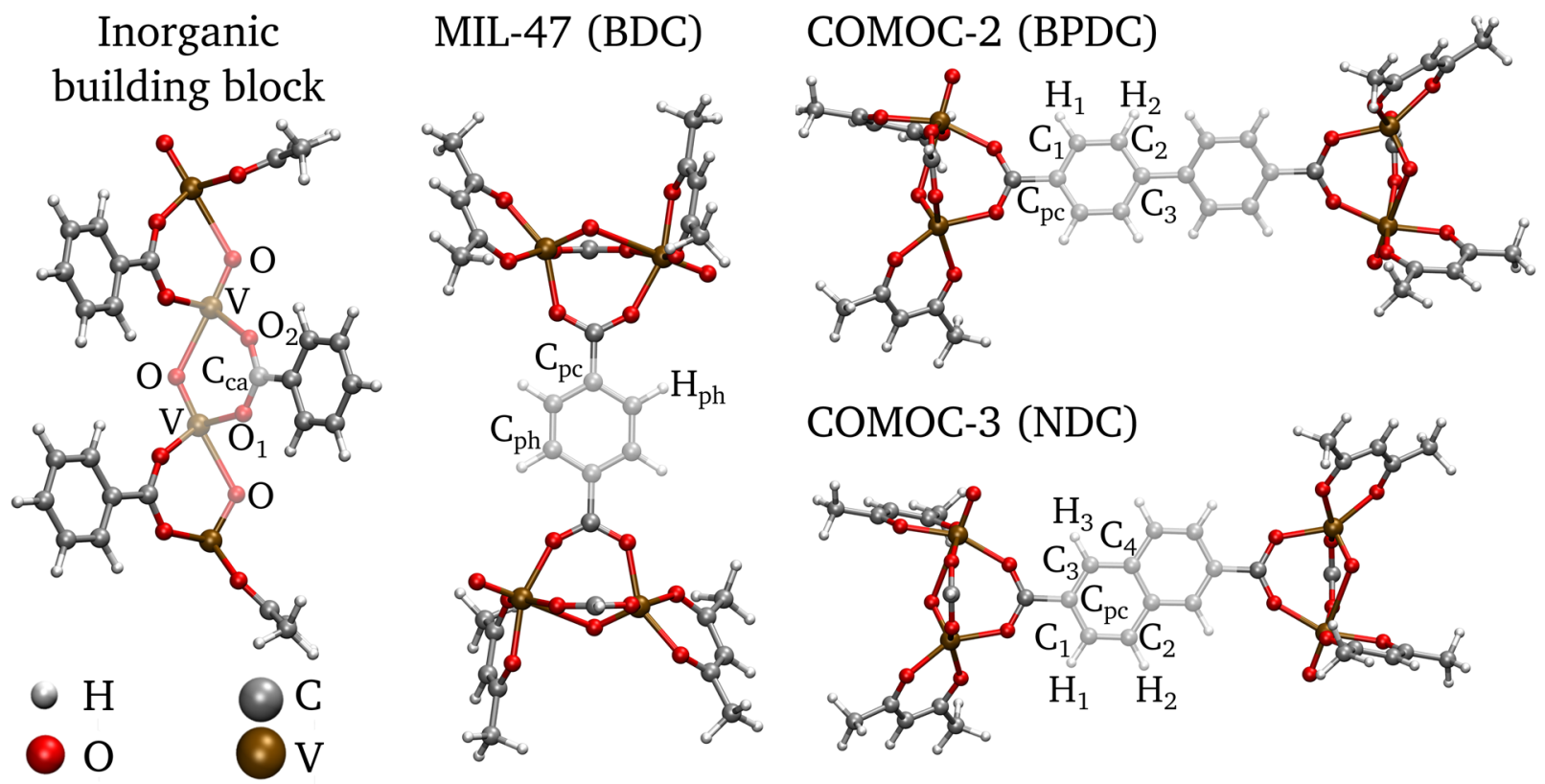

Figure 2. Overview of the model systems. The inorganic building block is used for the three MIL-47-type materials to describe the vanadium-oxide chain. The BDC, BPDC, and NDC building blocks are used in MIL-47, COMOC-2, and COMOC-3, respectively. The transparent region corresponds to the core region, and the unique atom types are indicated.

\section{METHODOLOGY}

2.1. Force Field Development. The workflow to generate force fields with QuickFF may be briefly summarized as

1. Perform electronic structure calculations on model systems representative for the building blocks of the MOF.

2. Use the optimized geometry, Hessian, and, optionally, atomic charges as input for QuickFF.

3. Transfer the derived force field parameters of the building blocks to the periodic cell.

4. Optionally add a van der Waals model a posteriori.

More elaborate details about the parametrization protocol can be found in ref 24 .

2.1.1. Model Systems. In this work, DFT calculations are performed on several model systems representing the different parts of MIL-47, COMOC-2, and COMOC-3 to generate the required first-principles input data. A single inorganic cluster model is proposed to model the vanadium-oxide chain in all three MOFs, while for each organic linker (BDC, BPDC, and NDC) a separate cluster is built. The model systems in this work are inspired by the ones for the MIL-53(Al) force field by Vanduyfhuys et al. ${ }^{72}$ The strong similarities between MIL-53 and MIL-47 suggest analogous cluster models for the inorganic part and organic linker, but the presence of asymmetric $\mathrm{V}-\mathrm{O}$ bonds along the vanadium-oxide chain demands some crucial interventions in the description of the $\mathrm{V}-\mathrm{O}$ bond. This asymmetric pattern is forced by terminating only one side of the vanadium-oxide chain with an oxygen atom (see Figure 2). In this way, an alternating short and long bond is obtained, identical to the periodic structure. The other oxygens of the octahedral coordination environment of the outermost vanadium atoms are capped with an acetylacetone molecule instead of hydrogens. The final cluster models have a neutral charge and are shown in Figure 2. They are optimized with a maximum spin of 2 (four vanadium atoms with one unpaired d- electron), as this yields the most reliable single-determinant description. ${ }^{18}$

2.1.2. Force Field Energy Expression. The force fields (FFs) are composed of two contributions: the covalent and the noncovalent interactions. The covalent force field terms (cov) describe the chemical bonds, while the noncovalent interactions (noncov) are the long-range interactions, and they are by definition separated into an electrostatic part (EI) and a part mimicking the van der Waals interactions (vdW). The multidimensional potential energy surface is then approximated with the following expression:

$$
V^{\mathrm{FF}}=\underbrace{V_{\text {bond }}+V_{\text {bend }}+V_{\text {oopd }}+V_{\text {torsion }}}_{V_{\mathrm{cov}}^{\mathrm{FF}}}+\underbrace{V_{\mathrm{EI}}+V_{\mathrm{vdW}}}_{V_{\text {noncov }}^{\mathrm{FF}}}
$$

The covalent interactions include harmonic bonds (bond), harmonic bends (bend), harmonic out-of-plane distances (oopd), and cosine dihedrals (torsion). All unknown parameters related to these expressions are estimated with QuickFF. The electrostatic interactions are modeled with a Coulombic potential between Gaussian charge distributions, and all interactions are included; that is, there are no exclusion rules. The atomic charges are derived from the DFT electron density of each model system via the MBIS procedure, a new iterative variant of the Hirshfeld atom-in-molecules (AIM) scheme. ${ }^{73}$ The radii of the Gaussian charge distributions are determined with the method proposed by Chen and Martínez. $^{74}$

The MM3-Buckingham potential ${ }^{75}$ is used to describe the van der Waals interactions.

$$
V(r)=\epsilon_{i j}\left[1.84 \times 10^{5} \exp \left(-12 \frac{r}{\sigma_{i j}}\right)-2.25\left(\frac{\sigma_{i j}}{r}\right)^{6}\right]
$$

The two parameters $\sigma_{i j}$ and $\epsilon_{i j}$ are the equilibrium distance and the energy parameter of the potential, respectively, and are 


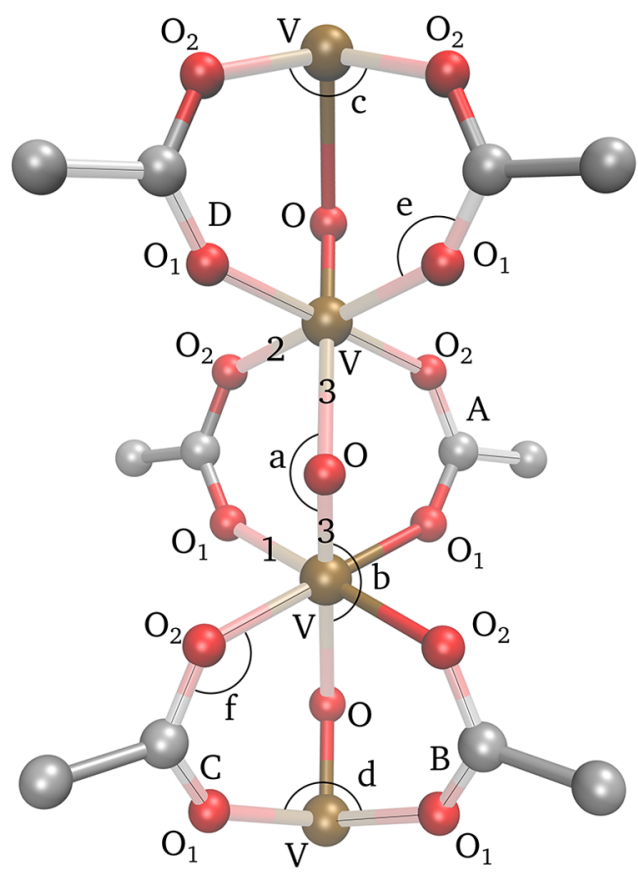

$\begin{array}{lll}\text { Bonds } & \text { Bends } & \text { Torsions } \\ \text { 1: }{\mathrm{V}-\mathrm{O}_{1}} & \text { a: } \mathrm{V}-\mathrm{O}-\mathrm{V} & \text { A: } \mathrm{V}-\mathrm{O}_{2}-\mathrm{C}_{\mathrm{ca}}-\mathrm{C}_{\mathrm{pc}} \\ \text { 2: } \mathrm{V}-\mathrm{O}_{2} & \text { b: } \mathrm{O}-\mathrm{V}-\mathrm{O} & \text { B: } \mathrm{V}-\mathrm{O}_{1}-\mathrm{C}_{\mathrm{ca}}-\mathrm{O}_{2} \\ \text { Urey-Bradley } & \text { c: } \mathrm{O}_{2}-\mathrm{V}-\mathrm{O}_{2} & \text { C: } \mathrm{V}-\mathrm{O}_{2}-\mathrm{C}_{\mathrm{ca}}-\mathrm{O}_{1} \\ \text { a: } \mathrm{V}-\mathrm{O}-\mathrm{V} & \text { d: } \mathrm{O}_{1}-\mathrm{V}-\mathrm{O}_{1} & \text { D: } \mathrm{V}-\mathrm{O}_{1}-\mathrm{C}_{\mathrm{ca}}-\mathrm{C}_{\mathrm{pc}} \\ \text { b: O-V-O } & \text { e: } \mathrm{V}-\mathrm{O}_{1}-\mathrm{C}_{\mathrm{ca}} & \\ & \text { f: } \mathrm{V}-\mathrm{O}_{2}-\mathrm{C}_{\mathrm{ca}} & \end{array}$

\section{Double-well potential}

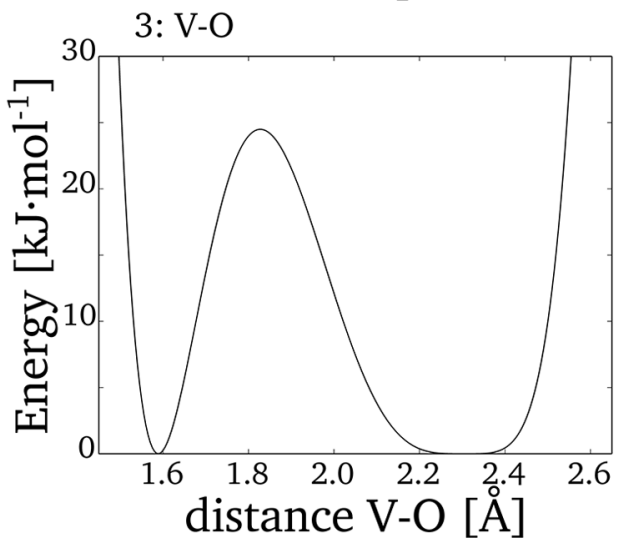

Figure 3. Overview of all covalent force field terms describing the inorganic chain. The double-well potential is shown on the right.

determined with empirical mixing rules for the interaction between atom $i$ and atom $j$ :

$$
\sigma_{i j}=\mathrm{s}_{\sigma} \cdot\left(\sigma_{i}+\sigma_{j}\right) \text { and } \epsilon_{i j}=\mathrm{s}_{\epsilon} \cdot\left(\sqrt{\epsilon_{i} \epsilon_{j}}\right)
$$

The interactions between the $1-2$ and $1-3$ bonded pairs are excluded following the MM3 rules, together with the 1-4 interactions as they were found to be too repulsive in the inorganic part of the MOFs under study, causing discrepancies in the reproduction of the correct unit cell shape. A possible reason is that the MM3 parameters have been fitted in environments that are not as dense as in the inorganic bricks encountered in MOFs. To compensate for this deficiency, slight modifications of the exclusion rule and the parameter values (section 4.2) are justified. Therefore, we define two uniform scaling parameters $\mathrm{s}_{\sigma}$ and $\mathrm{s}_{\epsilon}$ to slightly modify the values from the literature. The atomic $\epsilon_{i}$ and $\sigma_{i}$ parameters are taken from refs 75 and 76. This part is added to the force field after the QuickFF procedure has been applied, as it is assumed that these contributions are not present in the DFT calculations on the building blocks. This is indeed the case here, as an exchangecorrelation functional has been employed that includes only Pauli repulsion but no long-range dispersion interactions (vide infra).

The force field atom types used in the structure are indicated on the core region of the model systems (see Figure 2). The difference between the force fields for MIL-47, COMOC-2, and COMOC- 3 are thus the parameters related to the organic linker. Hence, the force field parameters extracted from the inorganic model system remain unaffected, comparable to synthesizing a MOF by combining the same inorganic salt with different carboxylic acids. Two different, alternating atom types $\left(\mathrm{O}_{1}\right.$ and $\left.\mathrm{O}_{2}\right)$ are assigned to the oxygens in the organic linkers, and both have different force field parameters. This is found to be necessary to accurately model the asymmetry arising because of the strong and weak bonds along the vanadium-oxide chain.
The force field parameters of the atoms inside the core region are transferred following the approach in ref 72 . An overview of all force field parameters is provided in the Supporting Information.

2.1.3. Extending QuickFF with MOF-Specific Force Field Terms. Some unique molecular environments in MOFs require more specific analytical expressions to adequately model all structural features. The MIL-47-type materials are typical examples where this is necessary because of the presence of the vanadium-oxide chain. The standard QuickFF protocol has been designed to give a first reasonable force field, but it can easily be modified with specific terms as illustrated hereafter.

The asymmetric $1 \mathrm{D}$ vanadium-oxide chain in MIL-47 cannot be described with a harmonic potential term, which has only one minimum, because the $\mathrm{V}-\mathrm{O}$ bond clearly has two distinct minima (see also Figure 3). To reproduce this pattern with a force field, the $\mathrm{V}-\mathrm{O}$ bond term is represented by a double-well potential with minima corresponding with the two equilibrium distances. Both a short (strong) and a long (weak) V-O bond are included in the core region of the inorganic model system (see Figure 2), and both bonds are approximated separately with a harmonic term in the QuickFF fitting procedure. This results in the corresponding force constants and rest values, which are used as input data to construct the double-well potential, satisfying the following conditions:

1. The potential energy has local minima both at the short $\left(r_{1}\right)$ and long $\left(r_{2}\right)$ bond distances.

2. The second-order derivative of the potential energy with respect to the bond distance in $r_{1}$ and $r_{2}$ corresponds with the determined force constants.

It is found via QuickFF that the weak bond has a zero force constant. The lowest-order polynomial approximation satisfying these conditions is given by 
Table 1. Comparison of Some Key Parameters for MIL-47(V) Obtained in This Work ${ }^{a}$ and by Bogaerts et al. ${ }^{38}$ with Periodic DFT Calculations in the Ferromagnetic (FM) and Antiferromagnetic (AFM) Spin Configuration ${ }^{b}$

\begin{tabular}{|c|c|c|c|c|c|}
\hline & \multicolumn{2}{|c|}{ force field - periodic } & \multirow{2}{*}{$\frac{\text { DFT - cluster }}{\text { B3LYP(FM) }}$} & \multicolumn{2}{|c|}{ DFT - periodic ${ }^{38}$} \\
\hline & excl. vdW & incl. vdW & & $\mathrm{PBE}+\mathrm{D} 3(\mathrm{FM})$ & $\mathrm{PBE}+\mathrm{D} 3(\mathrm{AFM})$ \\
\hline \multicolumn{6}{|c|}{ bond lengths $[\AA]$} \\
\hline $\mathrm{V}-\mathrm{V}$ & 3.524 & 3.481 & 3.531 & 3.435 & 3.441 \\
\hline $\mathrm{V}=\mathrm{O}$ & 1.594 & 1.592 & 1.588 & 1.655 & 1.652 \\
\hline $\mathrm{V}-\mathrm{O}$ & 2.313 & 2.305 & 2.311 & 2.090 & 2.106 \\
\hline $\mathrm{V}-\mathrm{O}_{\mathrm{i}}$ & $1.988 / 2.038$ & $1.983 / 2.028$ & $1.977 / 2.033$ & $1.992 / 2.001$ & $1.981 / 1.985$ \\
\hline $\mathrm{O}_{\mathrm{i}}-\mathrm{C}_{\mathrm{ca}}$ & $1.256 / 1.274$ & $1.255 / 1.273$ & $1.257 / 1.274$ & $1.277 / 1.278$ & $1.278 / 1.280$ \\
\hline $\mathrm{C}_{\mathrm{ca}}-\mathrm{C}_{\mathrm{pc}}$ & 1.489 & 1.504 & 1.493 & 1.489 & 1.487 \\
\hline $\mathrm{C}_{\mathrm{pc}}-\mathrm{C}_{\mathrm{ph}}$ & 1.399 & 1.403 & 1.399 & 1.404 & 1.404 \\
\hline $\mathrm{C}_{\mathrm{ph}}-\mathrm{C}_{\mathrm{ph}}$ & 1.387 & 1.386 & 1.388 & 1.390 & 1.389 \\
\hline $\mathrm{C}_{\mathrm{ph}}-\mathrm{H}_{\mathrm{ph}}$ & 1.082 & 1.081 & 1.082 & 1.089 & 1.089 \\
\hline \multicolumn{6}{|c|}{ bend angles [deg] } \\
\hline $\mathrm{V}-\mathrm{V}-\mathrm{V}$ & 168.0 & 165.1 & 166.4 & 166.6 & 167.2 \\
\hline $\mathrm{V}-\mathrm{O}=\mathrm{V}$ & 127.9 & 125.6 & 128.8 & 132.7 & 132.3 \\
\hline $\mathrm{O}-\mathrm{V}=\mathrm{O}$ & 178.2 & 175.8 & 176.8 & 172.4 & 173.3 \\
\hline $\mathrm{V}-\mathrm{O}_{\mathrm{i}}-\mathrm{C}_{\mathrm{ca}}$ & $131.2 / 140.1$ & $130.8 / 141.5$ & $130.3 / 141.7$ & $130.5 / 137.7$ & $130.7 / 138.3$ \\
\hline $\mathrm{O}_{\mathrm{i}}-\mathrm{V}-\mathrm{O}_{\mathrm{i}}$ & $87.4 / 91.9$ & $91.9 / 95.5$ & $87.7 / 91.3$ & $88.9 / 92.2$ & $89.1 / 91.2$ \\
\hline $\mathrm{O}_{1}-\mathrm{C}_{\mathrm{ca}}-\mathrm{O}_{2}$ & 125.6 & 124.0 & 125.3 & 125.6 & 125.3 \\
\hline
\end{tabular}

${ }^{a}$ The MM3 van der Waals parameters are rescaled [see section $\left.4.2\left(s_{\epsilon}=0.92, s_{\sigma}=1.14\right)\right] .{ }^{b}$ The structures of Bogaerts et al. are taken from the Cambridge Structural Database ${ }^{99}$ (CCDC numbers 1402265 and 1402266).

$$
V_{\text {bond }}(r)=\frac{K}{2} \frac{\left(r-r_{1}\right)^{2}\left(r-r_{2}\right)^{4}}{\left(r_{1}-r_{2}\right)^{4}}
$$

with $K$ the curvature of the strong bond. Together with the double-well potential, two Urey-Bradley terms were added for the $\mathrm{O}-\mathrm{V}=\mathrm{O}$ and $\mathrm{V}-\mathrm{O}=\mathrm{V}$ bonds along the asymmetric chain only to avoid overfitting. These terms can be interpreted as constraints such that the structure has an alternating shortlong pattern of $\mathrm{V}-\mathrm{O}$ bonds. A Urey-Bradley term is a term accounting for angle bending and is modeled via a harmonic bond between $1-3$ neighbors:

$$
V_{\text {Urey-Bradley }}(r)=\frac{K}{2}\left(r-r_{0}\right)^{2}
$$

The Urey-Bradley force constants, $K$, and rest values, $r_{0}$, can be derived with QuickFF by adding $\mathrm{V}-\mathrm{V}$ and $\mathrm{O}-\mathrm{O}$ bonds in the initial topology. In Figure 3, an overview of all covalent force field terms describing the inorganic chain is given.

A second case where the basic covalent terms are not sufficient is in the construction of an accurate force field for COMOC-2. A simple cosine term cannot correctly describe the dihedral motion of the $\mathrm{C}_{2}-\mathrm{C}_{3}-\mathrm{C}_{3}-\mathrm{C}_{2}$ pattern of the BPDC linker. It is not possible to obtain energy minima at $\pm \phi_{0}$ and $\pm\left(\pi-\phi_{0}\right)$ with a cosine with period $\frac{2 \pi}{m}(m \in \mathbb{N})$ for a general $\phi_{0}$. The potential term used for the $\mathrm{C}_{2}-\mathrm{C}_{3}-\mathrm{C}_{3}-\mathrm{C}_{2}$ pattern is

$$
V_{\mathrm{C}_{2}-\mathrm{C}_{3}-\mathrm{C}_{3}-\mathrm{C}_{2}}(\phi)=\frac{K_{\phi}}{2}\left[\cos (2 \phi)-\cos \left(2 \phi_{0}\right)\right]^{2}
$$

with $\phi_{0}$ taken from the DFT optimized geometry, which here is $38.225^{\circ}$. The advantage of QuickFF is that such terms can easily be added in the force constant refinement step of the fitting procedure. $^{24}$

2.2. Characterization via $\left(N, V, \sigma_{\mathrm{a}}=0, T\right)$ MD Simulations. The volume, $V$, is chosen as the collective variable to study the structural flexibility of the MIL-47-type materials. Obtaining the free energy, $F$, as a function of the volume enables us to clearly quantify the relative stability of the $\mathrm{np}$ and $\mathrm{lp}$ states at a certain temperature $T$, because both phases have a well-separated volume. The $F(V)$ curve can be obtained via thermodynamic integration according to the scheme recently proposed by Rogge et al. ${ }^{70}$ There, the $\left(N, V, \boldsymbol{\sigma}_{\mathrm{a}}=\mathbf{0}, T\right)$ ensemble was introduced, in which the cell shape and instantaneous isotropic pressure can fluctuate freely, while the volume is kept fixed and the anisotropic contribution $\sigma_{\mathrm{a}}$ to the stress tensor is controlled by a barostat during the MD simulation. The ensembleaveraged isotropic pressure $\langle P\rangle$ is extracted from such a simulation, making it possible to construct the $P(V)$ profile by repeating the simulations at different volumes. These data are then fitted with a polynomial. ${ }^{70}$ A volume grid spacing of $5 \AA^{3}$ is considered, and the initial structures for each volume are obtained by selecting different snapshots from a $\left(N, P, \boldsymbol{\sigma}_{\mathrm{a}}=\mathbf{0}, T\right)$ MD simulation. Furthermore, from the $P(V)$ curve, the bulk modulus of the equilibrium structure can be determined with the following relation:

$$
B=-\left.V \frac{\mathrm{d} P}{\mathrm{~d} V}\right|_{P=0}
$$

Moreover, some features from the fitted curve can be compared directly to the available experimental and theoretical data for MIL-47. This will be used as a validation of the first-principles derived force field. It is also possible to study the influence of temperature on the relative phase stability and on mechanical properties, such as the bulk modulus, by generating these profiles at various temperatures.

\section{COMPUTATIONAL DETAILS}

The model systems for the building blocks of MIL-47, COMOC-2, and COMOC-3 are constructed with Zeobuilder. $^{77} \mathrm{VMD}^{78}$ was used to visualize the structures. The DFT calculations on these systems are performed with Gaussian $09^{79}$ using the B3LYP exchange-correlation functional ${ }^{80-83}$ and the $6-311 G(\mathrm{~d}, \mathrm{p})$ basis set. ${ }^{84-87}$ The model systems have a neutral charge and spin 2 (one d-electron on four vanadium atoms). The covalent force field parameters are generated with QuickFF, ${ }^{24}$ and the atomic charges are derived according to 
the MBIS partitioning scheme as implemented in Horton. ${ }^{73}$ All force field calculations are carried out with $\mathrm{Yaff}^{88}$ using a supercell consisting of two unit cells along the lattice direction $b$ (see Figure 1). The long-range interactions are computed as follows: an Ewald summation with a real-space cutoff of $15 \AA$, an $\alpha$ of $0.213 \AA^{-1}$, and a reciprocal space cutoff $k_{\max }$ of 0.320 $\AA^{-1}$ for the electrostatic interactions and a smooth cutoff at 15 $\AA$ for the van der Waals interactions. Normal mode analysis is performed with TAMkin. ${ }^{89}$ A Verlet time step of 0.5 fs is used in all $\left(N, P, \boldsymbol{\sigma}_{\mathrm{a}}=\mathbf{0}, T\right)$ and $\left(N, V, \boldsymbol{\sigma}_{\mathrm{a}}=\mathbf{0}, T\right) \mathrm{MD}$ simulations. The temperature is controlled with a Nosé-Hoover chain thermostat $^{0-92}$ containing three beads with a relaxation time of $0.1 \mathrm{ps}$, while the pressure is controlled with a Martyna-TuckermanTobias-Klein barostat ${ }^{93,94}$ using a relaxation time of 1 ps. These choices were found to be suitable for the isostructural MIL-53(Al) by Rogge et al. ${ }^{70}$ A 100 ps equilibration time was taken into account, followed by a 500 ps production run for the $\left(N, V, \boldsymbol{\sigma}_{\mathbf{a}}=\mathbf{0}, T\right)$ MD simulations.

\section{FORCE FIELD VALIDATION}

4.1. Geometry Optimization of MIL-47. To validate the force fields, we mainly concentrate on MIL-47, because this is the most studied structure and sufficient reference data are available for comparison. Some important structural features at $0 \mathrm{~K}$, obtained with geometry optimizations of the periodic structure using the force field, are tabulated in Table 1 and compared with the cluster models and published periodic DFT calculations. ${ }^{38}$ Bogaerts et al. ${ }^{38}$ presented an efficient methodology to directly compare theoretically predicted geometries for crystalline materials to experimental X-ray diffractograms. MIL47 was chosen as a case study, and is in this context highly relevant in this discussion. Periodic DFT calculations (PBE $+\mathrm{D} 3^{95-97}$ ) on MIL-47 reveal that the most stable structure corresponds with a displaced chain structure and an antiferromagnetic spin configuration (AFM). Its counterpart with a ferromagnetic spin configuration (FM) is almost $30 \mathrm{~kJ}$. $\mathrm{mol}^{-1}$ per unit cell higher in energy, but the resulting diffraction pattern agrees best with the experimental pattern. ${ }^{38} \mathrm{~A}$ preliminary version of the MIL-47 force field has already been used in the work of Bogaerts et al., but no structural force field terms were considered to account for the displacement of the atoms in the vanadium chain. The diffractograms produced by this force field do not show the peaks arising from the shifted chains, as could be expected, and show large similarities with those obtained from first-principles without displaced vanadium chains. The force field constructed in this work contains additional terms to better describe this displacement of the vanadium atoms. Notice that the DFT cluster calculations used for the parametrization of the force field parameters systematically used parallel spins for the $\mathrm{V}$ atoms and thus best resemble the ferromagnetic spin configuration.

A first-principles parametrized force field should at least reproduce the DFT-optimized model systems from which it is derived. The force field optimized structure without van der Waals contributions should be compared with the DFT cluster models employed in the fitting of the force field parameters to assess the performance of the covalent force field parameters generated with QuickFF. As shown in Table 1, a good agreement is observed, indicating that the QuickFF parameters are indeed satisfactory to describe the covalent interactions.

The same conclusion can be drawn from Table 1 by comparing the periodic DFT calculations ${ }^{38}$ (with inclusion of dispersion corrections) with our periodic force field result, including van der Waals interactions. The main discrepancy is the overestimation of the $\mathrm{V}-\mathrm{V}$ bond distance and the increased asymmetry along the vanadium chain (strong bond is shorter and weak bond is longer than expected). This is a consequence of the DFT inorganic cluster model, which also displays this asymmetry, and can to a large extent be ascribed to the usage of a finite model system. However, the long and short $\mathrm{V}-\mathrm{O}$ distances of the cluster model are still within the experimental range of typical vanadium-oxygen bonds (V=O, 1.55-1.75 ; $\mathrm{V}-\mathrm{O}, 2.1-2.6 \AA) .{ }^{98}$ Other differences with the periodic DFT result are the $\mathrm{O}_{1}-\mathrm{V}-\mathrm{O}_{1}$ and $\mathrm{O}_{2}-\mathrm{V}-\mathrm{O}_{2}$ bend angles, which define the pore opening angle $\theta$ (see Figure 1). However, as will be shown in section 4.3 , the potential energy surface is very flat along these coordinates. Globally, we can conclude that the cluster model captures the most important features of the periodic framework, and the force field optimized structure follows this trend. Additionally a comparison may be made with the experimental structure. For that reason, MD simulations in the $\left(N, P, \sigma_{\mathrm{a}}=\mathbf{0}, T\right)$ ensemble were carried out at $300 \mathrm{~K}$ and $1 \mathrm{bar}$ (see Table S2).

4.2. Pressure-Induced Breathing of MIL-47. Overall, one can conclude from the previous section that the force field is able to correctly model the microscopic MIL-47 structure. One of the main objectives of the force field is to accurately predict the flexibility features of the material, i.e., the macroscopic pressure-induced breathing. To that end, simulations are performed in the $\left(N, V, \boldsymbol{\sigma}_{\mathrm{a}}=\mathbf{0}, T\right)$ ensemble at $300 \mathrm{~K}$ following the scheme outlined in Methodology. The transition pressures from the $\mathrm{lp}$ to the $\mathrm{np}$ states and vice versa are important properties describing this behavior. The lp-to-np transition pressure is defined as the maximum pressure that can be applied to the material before it transforms to the np phase and is given by the local maximum of the $P(V)$ curve between the lp and np volume. Similarly, the np-to-lp transition pressure is the minimum pressure to which the applied pressure can be lowered before the np structure opens to the lp state. It is given by the local minimum pressure between the np and lp volume (see Figure 4). In Table 2, the transition pressures observed in this work are compared to pressure ranges reported in the literature. The experimentally observed broad range of transition pressures was ascribed by Yot et al. ${ }^{29}$ to the presence of crystallites with a wide distribution of sizes, each with a different critical stress to initiate the structural transformation. Various possible magnetic configurations of MIL-47 lead to the DFT range of transition pressures ${ }^{55}$ in Table 2 . They were obtained by constructing energy-volume profiles through periodic DFT calculations at different spin configurations.

Initial simulations revealed that the original MM3 values of the atomic van der Waals parameters should be slightly modified to obtain transition pressures similar to the experimental values. Two uniform scaling parameters are used: (1) $\mathrm{s}_{\epsilon}=0.92$, the uniform scaling factor for the $\epsilon_{i}$; (2) $\mathrm{s}_{\sigma}=1.14$, the uniform scaling of the $\sigma_{i}$. The same scaling factors will be consistently applied to the other MIL-47-type materials. Pressure-volume profiles of MIL-47 at different scaling parameters are provided in the Supporting Information to illustrate the sensitivity (Figure S4). A similar rescaling was found to be necessary for the MIL-53(Al) force field by Vanduyfhuys et al. ${ }^{72}$ to reproduce the first-principles relative stability of the $n p$ and $\mathrm{lp}$ phases. With our values, the lp-to-np transition pressure is $125 \mathrm{MPa}$ at $300 \mathrm{~K}$, while the np-to-lp transition pressure amounts to $56 \mathrm{MPa}$ at $300 \mathrm{~K}$, coinciding with the results by Yot et al. ${ }^{29}$ The simulated volume decrease 


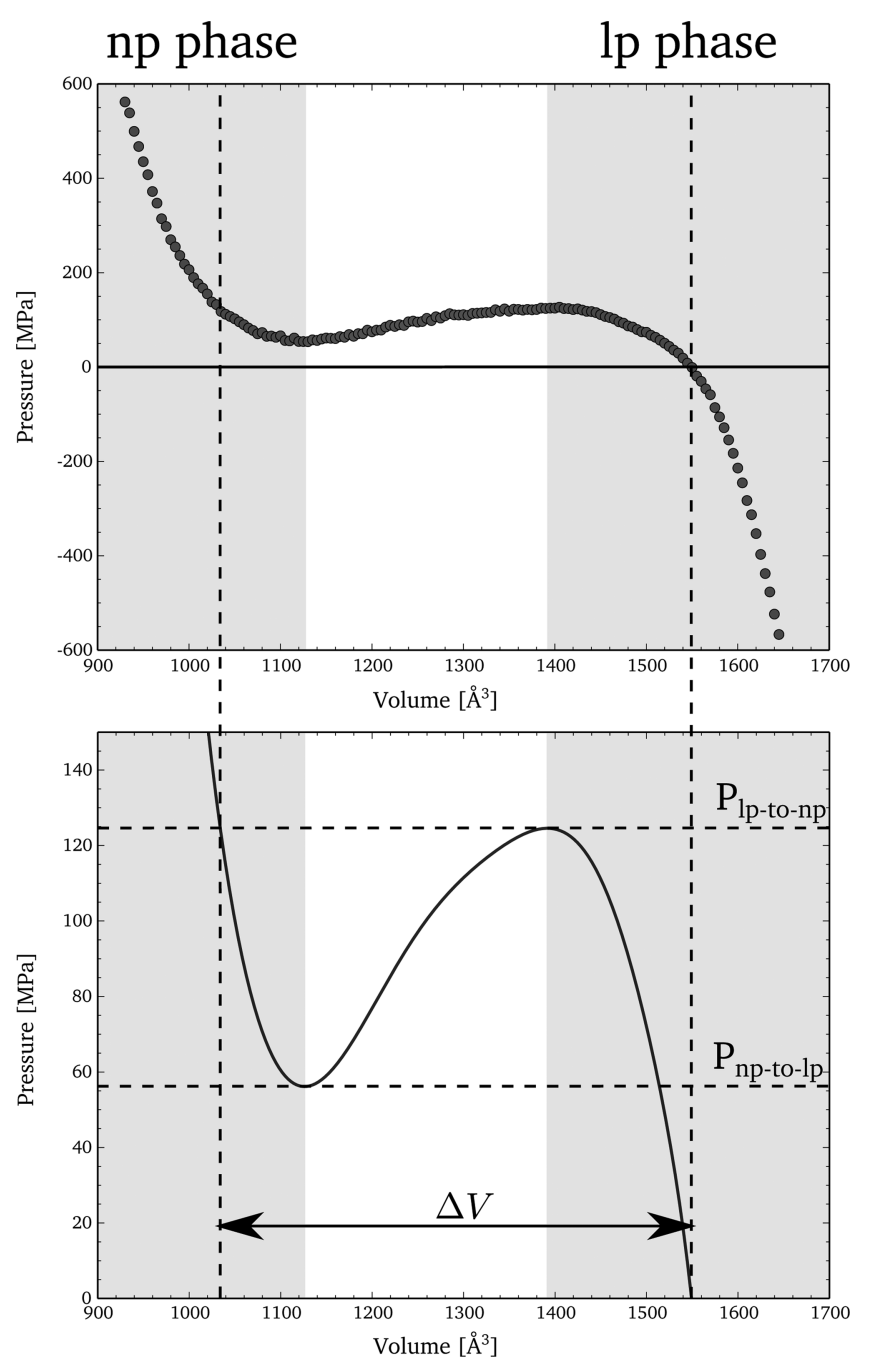

Figure 4. Resulting $P(V)$ curve of MIL-47 from the $\left(N, V, \boldsymbol{\sigma}_{\mathrm{a}}=\mathbf{0}, T\right) \mathrm{MD}$ simulations at $300 \mathrm{~K}$. The simulated pressure at each volume is indicated in the top pane. The polynomial fit is shown in a smaller pressure range in the bottom pane. The lp-to-np and np-to-lp transition pressures are indicated together with the $\Delta V / V_{\mathrm{lp}}$ volume contraction on the bottom figure. The np volume is defined here as the volume at the lp-to-np transition pressure in the $\mathrm{np}$ region.

Table 2. Overview of Transition Pressures for MIL-47

\begin{tabular}{|lcc|}
\multicolumn{1}{c}{ method } & $P_{\text {np-to-lp }}[\mathrm{MPa}]$ & $P_{\text {lp-to-np }}[\mathrm{MPa}]$ \\
\hline $\exp (300 \mathrm{~K})$ (ref 29) & $55-75$ & $85-125$ \\
$\exp (298 \mathrm{~K})$ (ref 61) & $58-95$ & $86-134$ \\
DFT (ref 55) & - & $82-124$ \\
FF MD (300 K) (ref 29) & 66 & 137 \\
FF MD (300 K) (this work) & 56 & 125
\end{tabular}

$\Delta V / V_{\mathrm{lp}}$ of $33 \%$, as indicated in Figure 4, is in agreement with the experimentally reported $38 \%$. Another experimental finding was the continuously decreasing volume of the $\mathrm{np}$ phase, instead of collapsing under higher pressure, which can be seen in the upper panel of Figure 4. Via X-ray diffraction, Yot et al. found a np volume of $947 \AA^{3}$ when applying a pressure of 178.1 $\mathrm{MPa}$. Using the $\left(N, P, \boldsymbol{\sigma}_{\mathrm{a}}=\mathbf{0}, T\right)$ ensemble, our force field predicts $1019(35) \AA^{3}$ at the same pressure and temperature. From the results presented in this section, it can be concluded that the force field is able to correctly mimic the pressure-induced flexibility of MIL-47.

4.3. Lattice Parameters of MIL-47, COMOC-2, and COMOC-3. A final test for the force field is the prediction of the lattice parameters. This is done for MIL-47, COMOC-2, and COMOC-3, for which experimental data are available. The definitions of the pore opening angle $\theta$ and the interdiagonal distance $D$, together with the $a, b$ and $c$ directions of the unit cell, are illustrated in Figure 1.

4.3.1. MIL-47. The lattice parameters predicted via periodic force field calculations (geometry optimization at $0 \mathrm{~K}$ ) are compared with the experimental (at room temperature) and DFT values (at $0 \mathrm{~K}$ ) in Table 3 . The most important difference is the underestimation of the pore opening angle $\theta$. The lattice parameter $a$ is overestimated, while $c$ is underestimated, and this is directly correlated to $\theta$. The overestimation of $b$ can be ascribed to the finite model system. However, the position of the minimum at $0 \mathrm{~K}$ is very sensitive to the precise parametrization of both the covalent and the van der Waals contributions, as the potential energy surface is very flat along the pore opening angle. In Figure 5, the outcome of a constrained potential energy scan at $0 \mathrm{~K}$ in the neighborhood of the lp minimum is shown, indicating energy differences of less than $2 \mathrm{~kJ} \cdot \mathrm{mol}^{-1}$ per unit cell between the pore opening angle of the force field equilibrium on the one hand and the experimental or DFT structures on the other hand. The lattice parameters corresponding with this structure are also indicated in Table 3. Given such flat potential energy surface, it is very difficult to exactly match experimental data with theoretical methods. In general, optimizations of flexible structures with theoretical methods pose a huge challenge, as was recently shown by some of the presenting authors. ${ }^{20}$

The symmetry breaking due to the displacement of the vanadium atoms perpendicular to the chain axis results in a lower energy (Figure S1). The structure without displacement, however, is also a stable minimum according to periodic DFT calculations. ${ }^{38}$ Both geometries are minima on the force field potential energy surface as confirmed by means of a normalmode analysis. The displaced chain is approximately $20 \mathrm{~kJ}$. $\mathrm{mol}^{-1}$ per unit cell more stable, and all our simulations are performed in this configuration. Recall that without addition of

Table 3. Comparison of Lattice Parameters for MIL-47(V)

\begin{tabular}{|c|c|c|c|c|c|c|c|c|c|c|}
\hline phase & method & $a[\AA]$ & $b[\AA]$ & $c[\AA]$ & $\alpha[\mathrm{deg}]$ & $\beta[\mathrm{deg}]$ & $\gamma[\mathrm{deg}]$ & $\theta[\mathrm{deg}]$ & $D[\AA]$ & $V\left[\AA^{3}\right]$ \\
\hline lp & $\exp ^{37 a}$ & 16.143 & 6.818 & 13.939 & 90.0 & 90.0 & 90.0 & 81.6 & 21.3 & 1534 \\
\hline $\mathrm{lp}$ & DFT $(F M)^{38}$ & 16.334 & 6.822 & 13.895 & 90.0 & 90.0 & 90.0 & 80.8 & 21.4 & 1548 \\
\hline $\mathrm{lp}$ & DFT $(\text { AFM })^{38}$ & 16.188 & 6.839 & 13.992 & 90.0 & 90.0 & 90.0 & 81.7 & 21.4 & 1549 \\
\hline lp & FF (this work) & 16.947 & 6.904 & 13.300 & 90.0 & 90.0 & 90.0 & 76.2 & 21.5 & 1556 \\
\hline $\mathrm{lp}$ & FF (this work) ${ }^{b}$ & 16.309 & 6.915 & 14.082 & 90.0 & 90.0 & 90.0 & $81.6^{b}$ & 21.5 & 1588 \\
\hline
\end{tabular}

${ }^{a}$ The experimental structure at room temperature by Barthelet et al. ${ }^{37}$ is also given. ${ }^{b}$ Geometry optimization with fixed pore opening angle $\theta$ at $81.6^{\circ}$. 


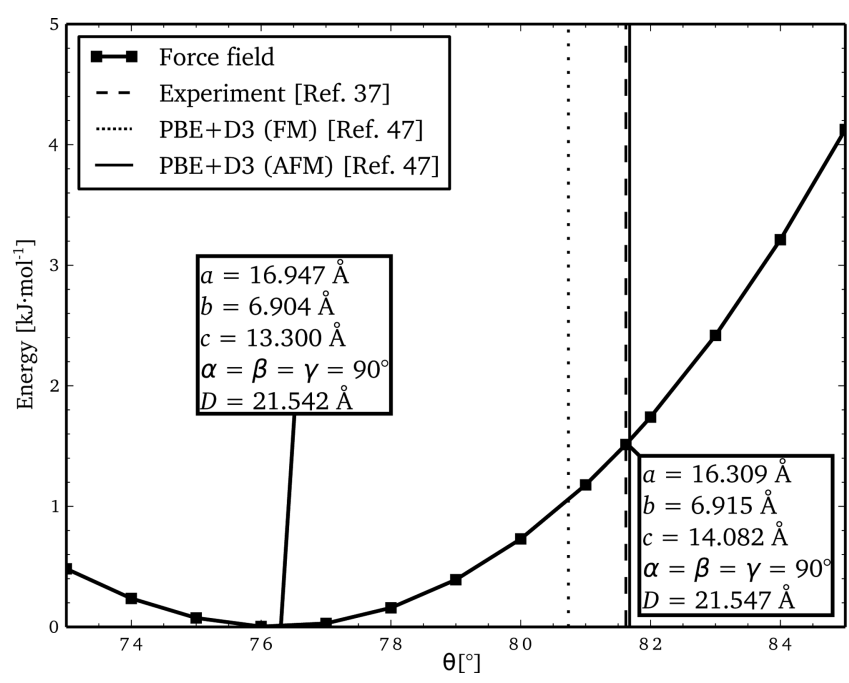

Figure 5. Scan of the pore opening angle in the lp phase of MIL-47 at $0 \mathrm{~K}$. The values obtained by Barthelet et al. ${ }^{37}$ and Bogaerts et al. ${ }^{38}$ are indicated. The unit cell parameters at the minima and under the constraint of the experimental pore-opening angle are also given.

the Urey-Bradley terms, the second minimum with displaced chains was not found. ${ }^{38}$

Note that for MIL-47 only a stable lp phase is reported at ambient conditions. With theoretical calculations both from DFT and from force field simulations it is possible to find a local minimum at the np phase on the potential energy surface at $0 \mathrm{~K}$. Structural parameters are taken up in the Supporting Information but not explicitly compared here because of the lack of experimental data.

4.3.2. COMOC-2. Highly accurate structural information on the empty COMOC-2 at low temperatures is not available. However, for COMOC-2, two structures, a lp and a np phase, were reported experimentally. ${ }^{40,44,45}$ Their geometric characteristics are given in Table 4. The np phase was found to be of monoclinic symmetry, whereas the lp corresponds to an orthorhombic unit cell. The results at $233 \mathrm{~K}$ were extracted by Liu et al. $^{40}$ from synchrotron XRD patterns under a certain $\mathrm{CO}_{2}$ pressure, which hinders a direct comparison with our empty framework. They also reported a pure lp phase at $293 \mathrm{~K}$. However, this structure was poorly reproducible because of the extreme synthesis procedure and with a small amount of remaining free linkers inside the pores, such that also here one should be careful with interpreting the results. Furthermore, the theoretical results reported in ref 40 stem from periodic DFT calculations on COMOC-2 at the PBE+D2 level ${ }^{95,100}$ using only a single $k$-point approach. It was recently shown by
Vanpoucke et $a .^{20}$ that for flexible materials more $k$-points should be used; thus, the values from ref 40 should be regarded with caution. The computational lattice parameters obtained by Liu et al. are also given in Table 4. It should be mentioned that Liu et al. did not report the case of displaced vanadium chains, similar to MIL-47, while this effect was taken into account in our simulations.

Our estimate for the lp phase reproduces the experimental and theoretical structures quite well. Similar features are observed as for the MIL-47 material with an underestimation of the pore opening angle. In contrast, the experimentally observed np volume is substantially larger than the DFT and force field predictions. The force field result is in agreement with the DFT-predicted orthorhombic shape of the np phase, which could indicate that the presence of guest molecules lies at the origin of the experimentally observed transformation to the monoclinic structure.

Liu et al. also determined the energy difference between the $\mathrm{lp}$ and $\mathrm{np}$ states at $0 \mathrm{~K}$ and found a value of approximately 20 $\mathrm{kJ} \cdot \mathrm{mol}^{-1}$ per unit cell at the $\mathrm{PBE}+\mathrm{D} 2$ level, which agrees relatively well with the $26 \mathrm{~kJ} \cdot \mathrm{mol}^{-1}$ per unit cell resulting from our force field simulations. The theoretically hypothesized socalled overstretched $\mathrm{np}$ phase with a pore opening angle of $147.5^{\circ}$ is also detected with our force field. However, this phase is more than $100 \mathrm{~kJ} \cdot \mathrm{mol}^{-1}$ per unit cell less stable than the regular np state, which could explain why it has not been observed experimentally. Henceforth, we will thus discard the existence of this additional phase configuration.

4.3.3. COMOC-3. COMOC-3 is a nonporous metal-organic framework; that is, it remains in a np state (see Figure 1). Structural information is available only on COMOC-3(as) or $\mathrm{V}(\mathrm{OH})(\mathrm{NDC}) \cdot \mathrm{H}_{2} \mathrm{O},{ }^{41}$ which is tabulated in Table 5. A periodic force field geometry optimization correctly leads to a np phase with an opening angle of $37^{\circ}$ and a $\gamma$ angle of $104^{\circ}$.

Summarizing, we may conclude that our force fields succeed in reproducing the essential characteristics of MIL-47-type materials. Geometrically, some bond distances and lattice parameters are overestimated or underestimated, which can mostly be ascribed to the finite cluster models used for the construction of the $\mathrm{ab}$ initio input data. However, the required asymmetry of the $\mathrm{O}-\mathrm{V}=\mathrm{O}$ chain is included, which allows the generation of subtle differences such as the structure with the displaced vanadium chains. The pressure-induced breathing for the MIL-47 is reproduced, which forms a solid basis to characterize the flexibility of the other materials, COMOC-2 and COMOC-3, under study.

Table 4. Comparison of Lattice Parameters for COMOC-2(V)

\begin{tabular}{|c|c|c|c|c|c|c|c|c|c|c|}
\hline phase & method & $a[\AA]$ & $b[\AA]$ & $c[\AA]$ & $\alpha[\mathrm{deg}]$ & $\beta[\mathrm{deg}]$ & $\gamma[\mathrm{deg}]$ & $\theta[\mathrm{deg}]$ & $D[\AA ̊]$ & $V\left[\AA^{3}\right]$ \\
\hline \multirow[t]{4}{*}{ lp } & $\exp (293 K)^{a 40}$ & 21.443 & 6.957 & 20.570 & 90 & 90 & 90 & 87.6 & 29.7 & 3069 \\
\hline & $\exp (233 \mathrm{~K})^{b 40}$ & 23.087 & 6.776 & 18.897 & 90 & 90 & 90 & 78.6 & 29.8 & 2956 \\
\hline & $\mathrm{DFT}^{40}$ & 21.642 & 6.927 & 20.498 & 90.0 & 90.0 & 90.0 & 86.9 & 29.8 & 3073 \\
\hline & FF (this work) & 24.038 & 6.858 & 18.219 & 90.0 & 90.0 & 90.0 & 74.3 & 30.2 & 3003 \\
\hline \multirow[t]{3}{*}{ np } & $\exp (233 \mathrm{~K})^{b 40}$ & 27.94 & 6.651 & 10.735 & 90 & 97.1 & 90 & 42.0 & 29.9 & 1979 \\
\hline & $\mathrm{DFT}^{40}$ & 28.111 & 6.991 & 6.809 & 90.0 & 90.0 & 90.0 & 27.2 & 28.9 & 1338 \\
\hline & FF (this work) & 28.552 & 6.840 & 7.592 & 90.0 & 90.0 & 90.0 & 29.8 & 29.5 & 1483 \\
\hline
\end{tabular}

${ }^{a}$ Data of the experimental lp phase at $293 \mathrm{~K}$ was obtained from Rietveld structure refinement based on COMOC-2( $\left.\mathrm{V}^{+\mathrm{IV}}\right)$, but with a small amount of remaining free linkers. ${ }^{b}$ The experimental lp phase at $233 \mathrm{~K}$ corresponds to a structure under 15 bar of $\mathrm{CO}_{2}$, while the experimental np phase at $233 \mathrm{~K}$ corresponds to a structure under $7.5 \mathrm{bar}$ of $\mathrm{CO}_{2}$. 
Table 5. Comparison of Lattice Parameters for COMOC-3(V)

\begin{tabular}{|c|c|c|c|c|c|c|c|c|c|}
\hline method & $a[\AA]$ & $b[\AA]$ & $c[\AA]$ & $\alpha[\mathrm{deg}]$ & $\beta[\operatorname{deg}]$ & $\gamma[\mathrm{deg}]$ & $\theta[\mathrm{deg}]$ & $D[\AA]$ & $\mathrm{V}\left[\AA^{3}\right]$ \\
\hline $\exp (293 K)^{a 41}$ & 24.985 & 6.880 & 7.508 & 90.0 & 90.0 & 107.7 & 33.5 & 26.1 & 1212 \\
\hline FF (this work) & 24.838 & 6.875 & 8.296 & 90.0 & 90.0 & 103.6 & 36.9 & 26.2 & 1377 \\
\hline
\end{tabular}

${ }^{a_{T}}$ The experimental structure is the COMOC-3(as) or $\mathrm{V}(\mathrm{OH})(\mathrm{NDC}) \cdot \mathrm{H}_{2} \mathrm{O}$ because there is no structural information available of COMOC-3(V).
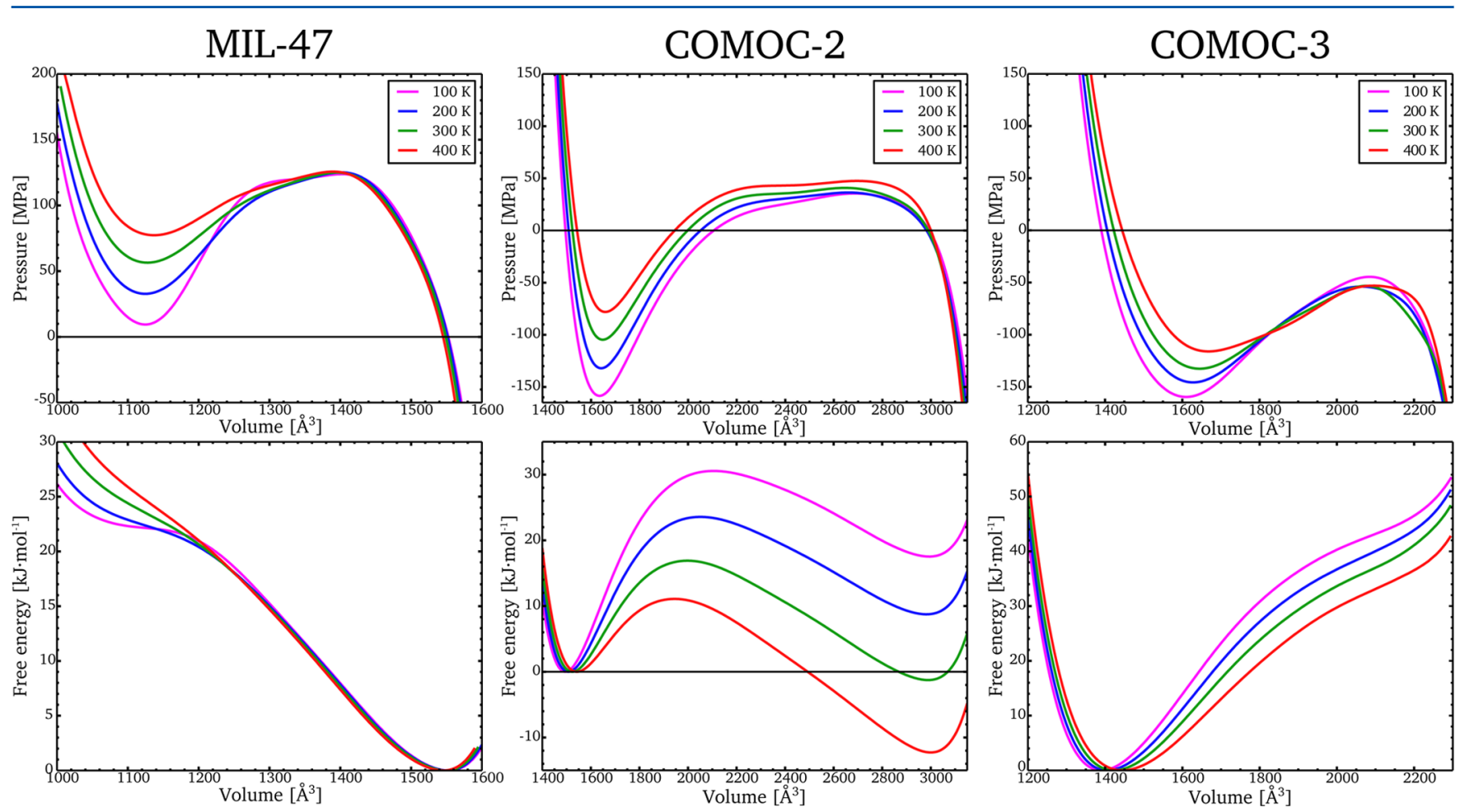

Figure 6. Fit of the $\left(N, V, \sigma_{\mathrm{a}}=0, T\right)$ MD data for MIL-47, COMOC-2, and COMOC-3 at temperatures ranging from 100 to $400 \mathrm{~K}$.
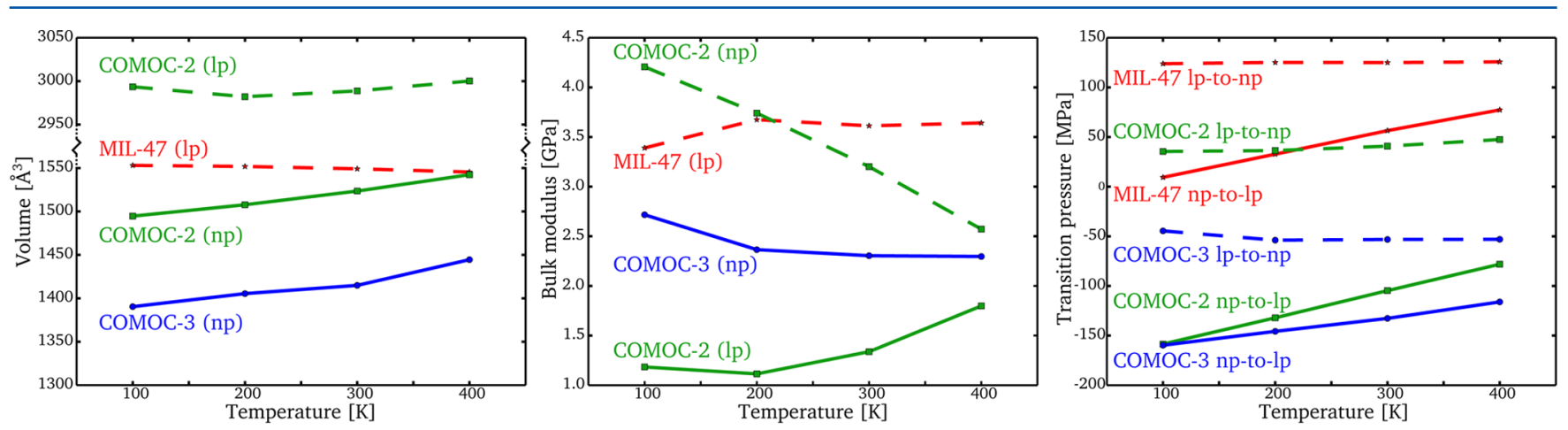

Figure 7. Influence of temperature on the volume, bulk moduli, and transition pressures of MIL-47-type materials.

\section{STRUCTURAL FLEXIBILITY IN MIL-47-TYPE MATERIALS}

Simulations in the $\left(N, V, \boldsymbol{\sigma}_{\mathrm{a}}=\mathbf{0}, T\right)$ ensemble were carried out with the force fields validated in the previous section. In this section we discuss the pressure versus volume and free energy versus volume profiles constructed for all three materials (see Figure 6). The simulations are carried out at various temperatures to investigate the influence of the temperature on derived characteristics such as the transition pressure and the bulk modulus. To the best of our knowledge such temperature behavior of the mechanical properties has not been studied before. These results are summarized in Figure 7.

The influence of the temperature on the $P(V)$ and $F(V)$ profiles is displayed in Figure 6. It is immediately clear that the temperature strongly affects the $\mathrm{np}-\mathrm{lp}$ transition pressure, which varies in MIL-47 almost linearly between 9 and $77 \mathrm{MPa}$ in the temperature range of $100-400 \mathrm{~K}$, while the reverse transition pressure does not seem to change substantially. This indicates that the hysteresis in the $P(V)$ curve decreases with increasing temperature. Analogously, it can be observed for the $F(V)$ curves that the free energy at low volumes increases with respect to the lp minimum with increasing temperatures. There is no np minimum at finite temperatures because no stable zero-pressure state is found at low volumes. Also, the negative thermal expansion (NTE) of the lp phase of MIL-47 can be seen in Figure 7. Indeed, the equilibrium volume, i.e., at zero external pressure, shifts toward smaller volumes with increasing temperature, in agreement with the experimental findings by 
Nanthamathee. ${ }^{101}$ An experimental volumetric thermal expansion coefficient, $\beta_{\mathrm{V}}$, of $-1.2 \times 10^{-5} \mathrm{~K}^{-1}$ was found by fitting the experimental $V(T)$ data in the temperature range of $12-$ $290 \mathrm{~K}^{101}$ to

$$
\beta_{\mathrm{V}}=\left(\frac{\partial \ln (V)}{\partial T}\right)_{P}
$$

Applying this procedure on our results reveals a simulated value of $-1.6 \times 10^{-5} \mathrm{~K}^{-1}$ for MIL-47 in the temperature range of $100-400 \mathrm{~K}$, which is in good agreement with the experimental result.

The shape of the $P(V)$ curve of COMOC-2 is shown in the middle pane of Figure 6, but the most crucial difference with MIL-47 is the range of the transition pressures. The lp-to-np transition pressure varies between 35-48 $\mathrm{MPa}$ depending on the temperature. The np-to-lp transition pressure even displays a larger range from $-159 \mathrm{MPa}$ to $-78 \mathrm{MPa}$. A negative pressure should be interpreted as an isotropic pulling effect on the material. The transition pressures corresponding with the structural transformations have not been directly measured via experiment. However, Couck et al. $^{44,45}$ applied a thermodynamic model (OFAST, developed by Coudert et al. ${ }^{62,63}$ ) to analyze their experimental data, consisting of adsorption and desorption isotherms of light hydrocarbons (C1, C2, and C3). This thermodynamic analysis enables the extraction of free energy differences between the empty host in the lp and the empty host in the np form $\left(\Delta F=F_{\mathrm{lp}}-F_{\mathrm{np}}\right)$. Values of about 40 $\mathrm{kJ} \cdot \mathrm{mol}^{-1}$ are found for temperatures around $300 \mathrm{~K}$. It is an indication that the $\mathrm{np}$ phase is much more stable than the $\mathrm{lp}$ phase at room temperature, which is not in full agreement with our simulations. Moreover, Liu et al. ${ }^{40}$ describe their COMOC$2\left(\mathrm{~V}^{\mathrm{IV}}\right)$ sample as containing a mixture of $\mathrm{np}$ and $\mathrm{lp}$ phases (powder X-ray diffraction at room temperature), which seems unlikely if the free energy difference between both phases is as large as $40 \mathrm{~kJ} \cdot \mathrm{mol}^{-1}$. Our results, displayed in Figure 6, indeed reveal a $\mathrm{lp}$ and $\mathrm{np}$ phase with a small free energy difference around $300 \mathrm{~K}$. It should also be noted that the isotherm fitting methodology, as applied by Couck et al., leads to phases of the material which are not strictly corresponding with the lp and np phases defined in this work and should rather be viewed as intermediate states at possibly different volumes. ${ }^{69}$

Liu et al. ${ }^{40}$ determined the energy difference between both phases as previously mentioned by means of periodic DFT calculations at the PBE+D2 level. A free energy scan as a function of the pore opening angle was also performed, thereby detecting three minima (np, lp, and overstretched np phases). They carried out frequency calculations enabling the computation of free energies. The modeled free energy profiles indicated that only at $650 \mathrm{~K}$ or higher does the lp phase become the most stable phase. This method, however, relies on the harmonic approximation, which is probably not the best choice for an intrinsic anharmonic material such as a flexible MOF. Our MD simulations, which include anharmonic effects, show that the lp phase already becomes the most stable structure around room temperature.

The free energy profile of COMOC-2 gives clear evidence for two stable minima of the empty frame, which are very close in free energy $\left(\sim 1 \mathrm{~kJ} \cdot \mathrm{mol}^{-1}\right.$ per unit cell in favor of the lp) at room temperature. Over the whole temperature range, the free energy difference $\left(\Delta F=F_{\mathrm{lp}}-F_{\mathrm{np}}\right)$ varies from 18 to $-12 \mathrm{~kJ}$. $\mathrm{mol}^{-1}$ per unit cell. This is the result of a complex balance between the amount of dispersive stabilization energy (favoring the $\mathrm{np}$ phase at low temperature) and the vibrational entropy (favoring the $\mathrm{lp}$ phase at high temperature). ${ }^{48}$ However, COMOC-2 displays no reversible temperature hysteresis behavior in this temperature range, which was observed for MIL-53(Al), ${ }^{47}$ because over the whole temperature range both phases remain minima of the free energy surface. The free energy profile also reveals a significant volume reduction of about 50\% between the $\mathrm{lp}$ and $\mathrm{np}$ structures, which is exceptionally high. This volume reduction combined with a relatively high lp-to-np transition pressure indicates that COMOC-2 could potentially be an attractive shock absorber. ${ }^{57}$ This is a consequence of a mechanically rigid lp phase due to the chemical nature of the vanadium chain and an increase of stabilizing dispersion interactions due to the extended BPDC linkers compared to the shorter BDC of MIL-47. The np phase displays positive thermal expansion (PTE) over the whole temperature range, while the $\mathrm{lp}$ phase has a more complex behavior (see Figure 7).

The third material under study is COMOC-3, for which a very stable $\mathrm{np}$ phase is found in agreement with experiment ${ }^{41}$ (see the right pane of Figure 6). In fact, the opposite behavior of MIL-47 is observed as both transition pressures are negative; hence, only a stable $\mathrm{np}$ state is obtained. Therefore, it is experimentally not possible to provoke structural transformations with a (positive) mechanical pressure as stimulus. This behavior can be understood by the predominance of stabilizing stacking interactions between two adjacent NDC linkers. The np-to-lp transition pressure is again temperaturedependent, but not as extremely as for COMOC-2 (see Figure 7 ), and the structure displays PTE similar to the np form of COMOC-2.

A final property that can be derived from these profiles is the bulk modulus and its dependence on the temperature (see Figure 7), which has been studied for only a very limited number of MOFs. ${ }^{53,102-104}$ Our simulations predict a MIL-47 bulk modulus of $3.6 \mathrm{GPa}$ at $300 \mathrm{~K}$, which remains constant between $200-400 \mathrm{~K}$. This order of magnitude is consistent with the fact that MIL-47 is considered to be more rigid than the similar framework materials MIL-53(Al) and MIL-53(Cr), which have reported experimental bulk moduli of $\sim 0.35 \mathrm{GPa}^{57}$ and $\sim 2 \mathrm{GPa}^{105}$ at room temperature. The $\mathrm{np}$ phase of COMOC-2 and COMOC-3 show bulk moduli which decrease with temperature, indicating a temperature-induced softening effect. This effect is the most pronounced for COMOC-2, where a decrease of the bulk modulus of almost $40 \%$ over a range of $300 \mathrm{~K}$ is found. Although the volume slightly expands, the bulk modulus of COMOC-2 in the lp form increases in the temperature range $200-400 \mathrm{~K}$. However, it still remains the material with the lowest resistance against mechanical deformation, probably due to the fact it has the longest linker. $^{102}$

\section{CONCLUSIONS}

We showed that the QuickFF ${ }^{24}$ parametrization protocol is able to generate force fields for stimuli-responsive MOFs. Herein we presented a systematic set of first-principles-derived force fields for the MIL-47-type materials, which are able to describe all structural features, characteristic to this family of MOFs, with inclusion of the breathing behavior under pressure. For these materials, some specific terms were added to correctly describe the asymmetry of the vanadium-oxide chains. Their performance has been validated on existing experimental and theoretical data available for the MIL-47-type materials. 
Subsequently, these force fields were used to study the structural flexibility of the empty framework. The three MIL47-type materials all display a different behavior, which can be understood in terms of increasing stabilizing dispersion interactions from MIL-47 to COMOC-3. While MIL-47 only has a stable lp phase, which requires a high pressure to undergo a structural transformation, COMOC- 3 resides in a very stable $\mathrm{np}$ phase. COMOC-2 is an intrinsic bistable MOF with lp and $\mathrm{np}$ minima, which are very sensitive to the temperature. The structural flexibility in empty MIL-47-type materials is thus determined by the host-host interactions, which is not so often encountered in other MOFs, as recently emphasized by Férey. ${ }^{13}$ As the flexibility strongly differs between the different MIL-47-type materials, our results underline the diversity of properties accessible by selecting the appropriate building blocks. To conclude, before one can assess the use of these stimuli-responsive MOFs in applications, a thorough understanding of the breathing behavior is required. The approach followed here opens up new perspectives to screen a larger set of materials and to select frameworks which behave rather as nanosprings, shock absorbers, or dampers.

\section{ASSOCIATED CONTENT}

\section{S Supporting Information}

The Supporting Information is available free of charge on the ACS Publications website at DOI: 10.1021/acs.jpcc.6b04422.

Force field parameters for the three materials, extra figures to distinguish the different MIL-47 structures, some key parameters and the lattice parameters of MIL47 using molecular dynamics simulations at the experimental conditions compared with published experimental data, and sensitivity of the pressure versus volume and free energy versus volume profiles on the van der Waals parameters (PDF)

\section{AUTHOR INFORMATION}

\section{Corresponding Author}

*E-mail: Veronique.VanSpeybroeck@UGent.be.

\section{Notes}

The authors declare no competing financial interest.

\section{ACKNOWLEDGMENTS}

This work is supported by the Fund for Scientific Research Flanders (FWO), the Research Board of Ghent University (BOF), and BELSPO in the frame of IAP/7/05. Funding was also received from the European Research Council under the European Community's Seventh Framework Programme [FP7 (2007-2013) ERC Grant Agreement 240483], and from the European Union's Horizon 2020 research and innovation programme [consolidator ERC Grant Agreement 647755 DYNPOR (2015-2020)]. Computational resources (Stevin Supercomputer Infrastructure) and services were provided by Ghent University. We also thank S. Vandenbrande and T. Verstraelen for their help in generating the force fields and $\mathrm{K}$. Lejaeghere for the insightful discussions on MIL-47.

\section{REFERENCES}

(1) Yaghi, O. M.; O’Keeffe, M.; Ockwig, N. W.; Chae, H. K.; Eddaoudi, M.; Kim, J. Reticular Synthesis and the Design of New Materials. Nature 2003, 423, 705-714.

(2) Kitagawa, S.; Kitaura, R.; Noro, S. Functional Porous Coordination Polymers. Angew. Chem., Int. Ed. 2004, 43, 2334-2375.
(3) Férey, G. Hybrid Porous Solids: Past, Present, Future. Chem. Soc. Rev. 2008, 37, 191-214.

(4) Czaja, A. U.; Trukhan, N.; Müller, U. Industrial Applications of Metal-Organic Frameworks. Chem. Soc. Rev. 2009, 38, 1284-1293.

(5) Zhou, H.-C.; Long, J. R.; Yaghi, O. M. Introduction to MetalOrganic Frameworks. Chem. Rev. 2012, 112, 673-674.

(6) Zhou, H.-C.; Kitagawa, S. Metal-Organic Frameworks (MOFs). Chem. Soc. Rev. 2014, 43, 5415-5418.

(7) Chang, Z.; Yang, D.-H.; Xu, J.; Hu, T.-L.; Bu, X.-H. Flexible Metal-Organic Frameworks: Recent Advances and Potential Applications. Adv. Mater. 2015, 27, 5432-5441.

(8) Horike, S.; Shimomura, S.; Kitagawa, S. Soft Porous Crystals. Nat. Chem. 2009, 1, 695-704.

(9) Férey, G.; Serre, C. Large Breathing Effects in ThreeDimensional Porous Hybrid Matter: Facts, Analyses, Rules and Consequences. Chem. Soc. Rev. 2009, 38, 1380-1399.

(10) Nagarkar, S. S.; Desai, A. V.; Ghosh, S. K. Stimulus-Responsive Metal-Organic Frameworks. Chem. - Asian J. 2014, 9, 2358-2376.

(11) Schneemann, A.; Bon, V.; Schwedler, I.; Senkovska, I.; Kaskel, S.; Fischer, R. A. Flexible Metal-Organic Frameworks. Chem. Soc. Rev. 2014, 43, 6062-6096.

(12) Coudert, F.-X. Responsive Metal-Organic Frameworks and Framework Materials: Under Pressure, Taking the Heat, in the Spotlight, with Friends. Chem. Mater. 2015, 27, 1905-1916.

(13) Férey, G. Structural Flexibility in Crystallized Matter: From History to Applications. Dalton Trans. 2016, 45, 4073-4089.

(14) Furukawa, H.; Cordova, K. E.; O’Keeffe, M.; Yaghi, O. M. The Chemistry and Applications of Metal-Organic Frameworks. Science 2013, 341, 1230444.

(15) Wilmer, C. E.; Leaf, M.; Lee, C. Y.; Farha, O. K.; Hauser, B. G.; Hupp, J. T.; Snurr, R. Q. Large-Scale Screening of Hypothetical MetalOrganic Frameworks. Nat. Chem. 2012, 4, 83-89.

(16) Yang, Q.; Liu, D.; Zhong, C.; Li, J.-R. Development of Computational Methodologies for Metal-Organic Frameworks and Their Application in Gas Separations. Chem. Rev. 2013, 113, 82618323.

(17) Colón, Y. J.; Snurr, R. Q. High-Throughput Computational Screening of Metal-Organic Frameworks. Chem. Soc. Rev. 2014, 43, 5735-5749.

(18) Odoh, S. O.; Cramer, C. J.; Truhlar, D. G.; Gagliardi, L. Quantum-Chemical Characterization of the Properties and Reactivities of Metal-Organic Frameworks. Chem. Rev. 2015, 115, 6051-6111.

(19) Coudert, F.-X.; Fuchs, A. H. Computational Characterization and Prediction of Metal-Organic Framework Properties. Coord. Chem. Rev. 2016, 307, 211-236.

(20) Vanpoucke, D. E. P.; Lejaeghere, K.; Van Speybroeck, V.; Waroquier, M.; Ghysels, A. Mechanical Properties from Periodic Plane Wave Quantum Mechanical Codes: the Challenge of the Flexible Nanoporous MIL-47(V) Framework. J. Phys. Chem. C 2015, 119, 23752-23766.

(21) Bureekaew, S.; Amirjalayer, S.; Tafipolsky, M.; Spickermann, C.; Roy, T. K.; Schmid, R. MOF-FF - A Flexible First-Principles Derived Force Field for Metal-Organic Frameworks. Phys. Status Solidi B 2013, 250, 1128-1141.

(22) Addicoat, M. A.; Vankova, N.; Akter, I. F.; Heine, T. Extension of the Universal Force Field to Metal-Organic Frameworks. J. Chem. Theory Comput. 2014, 10, 880-891.

(23) Bristow, J. K.; Tiana, D.; Walsh, A. Transferable Force Field for Metal-Organic Frameworks from First-Principles: BTW-FF. J. Chem. Theory Comput. 2014, 10, 4644-46521.

(24) Vanduyfhuys, L.; Vandenbrande, S.; Verstraelen, T.; Schmid, R.; Waroquier, M.; Van Speybroeck, V. QuickFF: A Program for a Quick and Easy Derivation of Force Fields for Metal-Organic Frameworks from Ab Initio Input. J. Comput. Chem. 2015, 36, 1015-1027.

(25) Salles, F.; Ghoufi, A.; Maurin, G.; Bell, R. G.; Mellot-Draznieks, C.; Férey, G. Molecular Dynamics Simulations of Breathing MOFs: Structural Transformations of MIL-53(Cr) Upon Thermal Activation of $\mathrm{CO}_{2}$ Adsorption. Angew. Chem., Int. Ed. 2008, 47, 8487-8491. 
(26) Coombes, D. S.; Corà, F.; Mellot-Draznieks, C.; Bell, R. G. Sorption-Induced Breathing in the Flexible Metal-Organic Framework CrMIL-53: Force-Field Simulations and Electronic Structure Analysis. J. Phys. Chem. C 2009, 113, 544-552.

(27) Grosch, J. S.; Paesani, F. Molecular-Level Characterization of the Breathing Behavior of the Jungle-Gym-Type DMOF-1 MetalOrganic Framework. J. Am. Chem. Soc. 2012, 134, 4207-4215.

(28) Hu, Z.; Zhang, L.; Jiang, J. Development of a Force Field for Zeolitic Imidazolate Framework-8 with Structural Flexibility. J. Chem. Phys. 2012, 136, 244703.

(29) Yot, P.; Ma, Q.; Haines, J.; Yang, Q.; Ghoufi, A.; Devic, T.; Serre, C.; Dmitriev, G.; Férey, G.; Zhong, G. L.; et al. Large breathing of the MOF MIL-47 $\left(\mathrm{V}^{I V}\right)$ Under Mechanical Pressure: A Joint Experimental-Modelling Exploration. Chem. Sci. 2012, 3, 1100-1104. (30) Zheng, B.; Sant, M.; Demontis, P.; Suffritti, G. B. Force Field for Molecular Dynamics Computations in Flexible ZIF-8 Framework. J. Phys. Chem. C 2012, 116, 933-938.

(31) Ortiz, A. U.; Boutin, A.; Fuchs, A. H.; Coudert, F.-X. Investigating the Pressure-Induced Amorphization of Zeolitic Imidazolate Framework ZIF-8: Mechanical Instability Due to Shear Mode Softening. J. Phys. Chem. Lett. 2013, 4, 1861-1865.

(32) Zhang, L.; Hu, Z.; Jiang, J. Sorption-Induced Structural Transition of Zeolitic Imidazolate Framework-8: A Hybrid Molecular Simulation Study. J. Am. Chem. Soc. 2013, 135, 3722-3728.

(33) Wu, X.; Huang, J.; Cai, W.; Jaroniec, M. Force Field for ZIF-8 Flexible Frameworks: Atomistic Simulation of Adsorption, Diffusion of Pure Gases as $\mathrm{CH}_{4}, \mathrm{H}_{2}, \mathrm{CO}_{2}$ and $\mathrm{N}_{2}$. RSC Adv. 2014, 4, 16503-16511.

(34) Rappe, A. K.; Casewit, C. J.; Colwell, K. S.; Goddard, W. A., III; Skiff, W. M. UFF, a Full Periodic Table Force Field for Molecular Mechanics and Molecular Dynamics Simulations. J. Am. Chem. Soc. 1992, 114, 10024-10035.

(35) Dauber-Osguthorpe, P.; Roberts, V. A.; Osguthorpe, D. J.; Wolff, J.; Genest, M.; Hagler, A. T. Structure and Energetics of Ligand Binding to Proteins: Escherichia Coli Dihydrofolate ReductaseTrimethoprim, a Drug-Receptor System. Proteins: Struct., Funct., Genet. 1988, 4, 31-47.

(36) Wang, J.; Wolf, R. M.; Caldwell, J. W.; Kollman, P. A.; Case, D. A. Development and Testing of a General Amber Force Field. J. Comput. Chem. 2004, 25, 1157-1174.

(37) Barthelet, K.; Marrot, J.; Riou, D.; Férey, G. A Breathing Hybrid Organic-Inorganic Solid with Very Large Pores and High Magnetic Characteristics. Angew. Chem., Int. Ed. 2002, 41, 281-284.

(38) Bogaerts, T.; Vanduyfhuys, L.; Vanpoucke, D. E. P.; Wieme, J.; Waroquier, M.; Van Der Voort, P.; Van Speybroeck, V. Fine-Tuning the Theoretically Predicted Structure of MIL-47(V) with the Aid of Powder X-Ray Diffraction. CrystEngComm 2015, 17, 8612-8622.

(39) Van Der Voort, P.; Leus, K.; Liu, Y.-Y.; Vandichel, M.; Van Speybroeck, V.; Waroquier, M.; Biswas, S. Vanadium Metal-Organic Frameworks: Structures and Applications. New J. Chem. 2014, 38, $1853-1867$.

(40) Liu, Y.-Y.; Couck, S.; Vandichel, M.; Grzywa, M.; Leus, K.; Biswas, S.; Volkmer, D.; Gascon, J.; Kapteijn, F.; Denayer, J. F. M.; et al. New V-IV-Based Metal-Organic Framework Having Framework Flexibility and High $\mathrm{CO}_{2}$ Adsorption Capacity. Inorg. Chem. 2013, 52, $113-120$.

(41) Liu, Y.-Y.; Leus, K.; Grzywa, M.; Weinberger, D.; Strubbe, K.; Vrielinck, H.; Van Deun, R.; Volkmer, D.; Van Speybroeck, V.; Van Der Voort, P. Synthesis, Structural Characterization, and Catalytic Performance of a Vanadium-Based Metal-Organic Framework (COMOC-3). Eur. J. Inorg. Chem. 2012, 2012, 2819-2827.

(42) Senkovska, I.; Hoffmann, F.; Fröba, M.; Getzschmann, J.; Böhlmann, W.; Kaskel, S. New Highly Porous Aluminium Based Metal-Organic Frameworks: $\mathrm{Al}(\mathrm{OH})$ (NDC) (NDC $=2,6-\mathrm{Naph}-$ thalene Dicarboxylate) and $\mathrm{Al}(\mathrm{OH})(\mathrm{BPDC})\left(\mathrm{BPDC}=4,4^{\prime}\right.$-Biphenyl Dicarboxylate). Microporous Mesoporous Mater. 2009, 122, 93-98.

(43) Lu, W.; Wei, Z.; Gu, Z.-Y.; Liu, T.-F.; Park, J.; Park, J.; Tian, J.; Zhang, M.; Zhang, Q.; Gentle, T., III; et al. Tuning the Structure and Function of Metal-Organic Frameworks via Linker Design. Chem. Soc. Rev. 2014, 43, 5561-5593.
(44) Couck, S.; Van Assche, T. R. C.; Liu, Y.-Y.; Baron, G. V.; Van Der Voort, P.; Denayer, J. F. M. Adsorption and Separation of Small Hydrocarbons on the Flexible, Vanadium-Containing MOF, COMOC-2. Langmuir 2015, 31, 5063-5070.

(45) Couck, S.; Van Assche, T. R. C.; Liu, Y.-Y.; Baron, G. V.; Van Der Voort, P.; Denayer, J. F. M. Correction to "Adsorption and Separation of Small Hydrocarbons on the Flexible, VanadiumContaining MOF, COMOC-2. Langmuir 2015, 31, 13467-13467.

(46) Leclerc, H.; Devic, T.; Devautour-Vinot, S.; Bazin, P.; Audebrand, N.; Férey, G.; Daturi, M.; Vimont, A.; Clet, G. Influence of the Oxidation State of the Metal Center on the Flexibility and Adsorption Properties of a Porous Metal Organic Framework: MIL47(V). J. Phys. Chem. C 2011, 115, 19828-19840.

(47) Liu, Y.; Her, J.-H.; Dailly, A.; Ramirez-Cuesta, A. J.; Neumann, D. A.; Brown, C. M. Reversible Structural Transition in MIL-53 with Large Temperature Hysteresis. J. Am. Chem. Soc. 2008, 130, 1181311818.

(48) Walker, A. M.; Civalleri, B.; Slater, B.; Mellot-Draznieks, C.; Corà, F.; Zicovich-Wilson, C. M.; Román-Pérez, G.; Soler, J. M.; Gale, J. D. Flexibility in a Metal-Organic Framework Controlled by Weak Dispersion Forces: the Bistability of MIL-53(Al). Angew. Chem., Int. Ed. 2010, 49, 7501-7503.

(49) Tan, J. C.; Cheetham, A. K. Mechanical Properties of Hybrid Inorganic-Organic Framework Materials: Establishing Fundamental Structure-Property Relationships. Chem. Soc. Rev. 2011, 40, 10591080.

(50) Ortiz, A. U.; Boutin, A.; Fuchs, A. H.; Coudert, F.-X. Anisotropic Elastic Properties of Flexible Metal-Organic Frameworks: How Soft Are Soft Porous Crystals? Phys. Rev. Lett. 2012, 109, 195502.

(51) Li, W.; Henke, S.; Cheetham, A. K. Research Update: Mechanical Properties of Metal-Organic Frameworks - Influence of Structure and Chemical Bonding. APL Mater. 2014, 2, 123902.

(52) Yang, K.; Zhou, G.; Xu, Q. The Elasticity of MOFs Under Mechanical Pressure. RSC Adv. 2016, 6, 37506-37514.

(53) Ortiz, A. U.; Boutin, A.; Fuchs, A. H.; Coudert, F.-X. MetalOrganic Frameworks with Wine-Rack Motif: What Determines Their Flexibility and Elastic Properties? J. Chem. Phys. 2013, 138, 174703.

(54) Heinen, J.; Dubbeldam, D. Understanding and Solving Disorder in the Substitution Pattern of Amino Functionalized MIL-47(V). Dalton Trans. 2016, 45, 4309-4315.

(55) Vanpoucke, D. E. P.; Jaeken, J. W.; De Baerdemacker, S.; Lejaeghere, K.; Van Speybroeck, V. Quasi-1D Physics in MetalOrganic Frameworks: MIL-47(V) from First Principles. Beilstein J. Nanotechnol. 2014, 5, 1738-1748.

(56) Beurroies, I.; Boulhout, M.; Llewellyn, P. L.; Kuchta, B.; Férey, G.; Serre, C.; Denoyel, R. Using Pressure to Provoke the Structural Transition of Metal-Organic Frameworks. Angew. Chem., Int. Ed. 2010, 49, 7526-7529.

(57) Yot, P. G.; Boudene, Z.; Macia, J.; Granier, D.; Vanduyfhuys, L.; Verstraelen, T.; Van Speybroeck, V.; Devic, T.; Serre, C.; Férey, G.; et al. Metal-Organic Frameworks as Potential Shock Absorbers: The Case of the Highly Flexible MIL-53(Al). Chem. Commun. 2014, 50, 9462-9464.

(58) Rodriguez, J.; Beurroies, I.; Loiseau, T.; Denoyel, R.; Llewellyn, P. L. The Direct Heat Measurement of Mechanical Energy Storage Metal-Organic Frameworks. Angew. Chem., Int. Ed. 2015, 54, 46264630.

(59) McKellar, S. C.; Moggach, S. A. Structural Studies of MetalOrganic Frameworks Under High Pressure. Acta Crystallogr., Sect. B: Struct. Sci., Cryst. Eng. Mater. 2015, 71, 587-607.

(60) Yot, P. G.; Vanduyfhuys, L.; Alvarez, E.; Rodriguez, J.; Itié, J.-P.; Fabry, P.; Guillou, N.; Devic, T.; Beurroies, I.; Llewellyn, P. L.; et al. Mechanical Energy Storage Performance of an Aluminum Fumarate Metal-Organic Framework. Chem. Sci. 2016, 7, 446-450.

(61) Rodriguez, J.; Beurroies, I.; Coulet, M.-V.; Fabry, P.; Devic, T.; Serre, C.; Denoyel, R.; Llewellyn, P. L. Thermodynamics of the Structural Transition in Metal-Organic Frameworks. Dalton Trans. 2016, 45, 4274-4282. 
(62) Coudert, F.-X.; Jeffroy, M.; Fuchs, A. H.; Boutin, A.; MellotDraznieks, C. Thermodynamics of Guest-Induced Structural Transitions in Hybrid Organic-Inorganic Frameworks. J. Am. Chem. Soc. 2008, 130, 14294-14302.

(63) Coudert, F.-X. The Osmotic Framework Adsorbed Solution Theory: Predicting Mixture Coadsorption in Flexible Nanoporous Materials. Phys. Chem. Chem. Phys. 2010, 12, 10904-10913.

(64) Coudert, F.-X.; Boutin, A.; Fuchs, A. H. A Thermodynamic Description of the Adsorption-Induced Structural Transitions in Flexible MIL-53 Metal-Organic Framework. Mol. Phys. 2014, 112, $1257-1261$.

(65) Neimark, A. V.; Coudert, F.-X.; Boutin, A.; Fuchs, A. H. StressBased Model for the Breathing of Metal-Organic Frameworks. J. Phys. Chem. Lett. 2010, 1, 445-449.

(66) Bousquet, D.; Coudert, F.-X.; Boutin, A. Free Energy Landscapes for the Thermodynamic Understanding of AdsorptionInduced Deformations and Structural Transitions in Porous Materials. J. Chem. Phys. 2012, 137, 044118.

(67) Bousquet, D.; Coudert, F.-X.; Fossati, A. G. J.; Neimark, A. V.; Fuchs, A. H.; Boutin, A. Adsorption Induced Transitions in Soft Porous Crystals: An Osmotic Potential Approach to Multistability and Intermediate Structures. J. Chem. Phys. 2013, 138, 174706.

(68) Ghysels, A.; Vanduyfhuys, L.; Vandichel, M.; Waroquier, M.; Van Speybroeck, V.; Smit, B. On the Thermodynamics of Framework Breathing: A Free Energy Model for Gas Adsorption in MIL-53. J. Phys. Chem. C 2013, 117, 11540-11554.

(69) Vanduyfhuys, L.; Ghysels, A.; Rogge, S. M. J.; Demuynck, R.; Van Speybroeck, V. Semi-Analytical Mean-Field Model for Predicting Breathing in Metal-Organic Frameworks. Mol. Simul. 2015, 41, 13111328.

(70) Rogge, S. M. J.; Vanduyfhuys, L.; Ghysels, A.; Waroquier, M.; Verstraelen, T.; Maurin, G.; Van Speybroeck, V. A Comparison of Barostats for the Mechanical Characterization of Metal-Organic Frameworks. J. Chem. Theory Comput. 2015, 11, 5583-5597.

(71) Kirkwood, J. G. Statistical Mechanics of Fluid Mixtures. J. Chem. Phys. 1935, 3, 300-313.

(72) Vanduyfhuys, L.; Verstraelen, T.; Vandichel, M.; Waroquier, M.; Van Speybroeck, V. Ab Initio Parametrized Force Field for the Flexible Metal-Organic Framework MIL-53(Al). J. Chem. Theory Comput. 2012, 8, 3217-3231.

(73) Verstraelen, T.; Boguslawski, K.; Tecmer, P.; Heidar-Zadeh, F.; Chan, M.; Kim, T. D.; Zhao, T.; Vandenbrande, S.; Yang, D.; González-Espinoza, C. E.; et al. Horton 2.0.0, 2015. http://theochem. github.com/horton/ (accessed June 9, 2016).

(74) Chen, J.; Martínez, T. J. QTPIE: Charge Transfer with Polarization Current Equalization. A Fluctuating Charge Model with Correct Asymptotics. Chem. Phys. Lett. 2007, 438, 315-320.

(75) Lii, J. H.; Allinger, N. L. Molecular Mechanics. The MM3 Force Field for Hydrocarbons. 3. The van der Waals Potentials and Crystal Data for Aliphatic and Aromatic Hydrocarbons. J. Am. Chem. Soc. 1989, 111, 8576-8582.

(76) Allinger, N. L.; Zhou, X.; Bergsma, J. Molecular Mechanics Parameters. J. Mol. Struct.: THEOCHEM 1994, 312, 69-83.

(77) Verstraelen, T.; Van Speybroeck, V.; Waroquier, M. ZEOBUILDER: A GUI Toolkit for the Construction of Complex Molecular Structures on the Nanoscale with Building Blocks. J. Chem. Inf. Model. 2008, 48, 1530-1541.

(78) Humphrey, W.; Dalke, A.; Schulten, K. VMD: Visual Molecular Dynamics. J. Mol. Graphics 1996, 14, 33-38.

(79) Frisch, M. J.; Trucks, G. W.; Schlegel, H. B.; Scuseria, G. E.; Robb, M. A.; Cheeseman, J. R.; Scalmani, G.; Barone, V.; Mennucci, B.; Petersson, G. A. et al. Gaussian09, revision E.01; Gaussian Inc.: Wallingford CT, 2009.

(80) Becke, A. D. Density-Functional Exchange-Energy Approximation with Correct Asymptotic Behavior. Phys. Rev. A: At., Mol., Opt. Phys. 1988, 38, 3098-3100.

(81) Becke, A. D. Density-Functional Thermochemistry. III. The Role of Exact Exchange. J. Chem. Phys. 1993, 98, 5648-5652.
(82) Lee, C.; Yang, W.; Parr, R. G. Development of the Colle-Salvetti Correlation-Energy Formula into a Functional of the Electron Density. Phys. Rev. B: Condens. Matter Mater. Phys. 1988, 37, 785-789.

(83) Vosko, S. H.; Wilk, L.; Nusair, M. Accurate Spin-Dependent Electron Liquid Correlation Energies for Local Spin Density Calculations: A Critical Analysis. Can. J. Phys. 1980, 58, 1200-1211.

(84) Wachters, A. J. H. Gaussian Basis Sets for Molecular Wavefunctions Containing Third-Row Atoms. J. Chem. Phys. 1970, 52, 1033-1036.

(85) Hay, P. J. Gaussian Basis Sets for Molecular Calculations. The Representation of 3d Orbitals in Transition-Metal Atoms. J. Chem. Phys. 1977, 66, 4377-4384.

(86) Krishnan, R.; Binkley, J. S.; Seeger, R.; Pople, J. A. SelfConsistent Molecular Orbital Methods. 20. Basis Set for Correlated wave-Functions. J. Chem. Phys. 1980, 72, 650-654.

(87) Raghavachari, K.; Trucks, G. W. Highly Correlated Systems. Excitation Energies of First Row Transition Metals Sc-Cu. J. Chem. Phys. 1989, 91, 1062-1065.

(88) Verstraelen, T.; Vanduyfhuys, L.; Vandenbrande, S.; Rogge, S. M. J. Yaff, yet another force field. http://molmod.ugent.be/software/ (accessed June 9, 2016).

(89) Ghysels, A.; Verstraelen, T.; Hemelsoet, K.; Waroquier, M.; Van Speybroeck, V. TAMkin: A Versatile Package for Vibrational Analysis and Chemical Kinetics. J. Chem. Inf. Model. 2010, 50, 1736-1750.

(90) Hoover, W. G. Canonical Dynamics: Equilibrium Phase-Space Distributions. Phys. Rev. A: At., Mol., Opt. Phys. 1985, 31, 1695-1697.

(91) Nosé, S. An Extension of the Canonical Ensemble Molecular Dynamics Method. Mol. Phys. 1986, 57, 187-191.

(92) Martyna, G. J.; Klein, M. L.; Tuckerman, M. E. Nosé-Hoover chains: The Canonical Ensemble via Continuous Dynamics. J. Chem. Phys. 1992, 97, 2635-2643.

(93) Martyna, G. J.; Tobias, D. J.; Klein, M. L. Constant Pressure Molecular Dynamics Algorithms. J. Chem. Phys. 1994, 101, 41774189.

(94) Martyna, G. J.; Tuckerman, M. E.; Tobias, D. J.; Klein, M. L. Explicit Reversible Integrators for Extended Systems Dynamics. Mol. Phys. 1996, 87, 1117-1157.

(95) Perdew, J. P.; Burke, K.; Ernzerhof, M. Generalized Gradient Approximation Made Simple. Phys. Rev. Lett. 1996, 77, 3865-3868.

(96) Grimme, S.; Antony, J.; Ehrlich, S.; Krieg, H. A Consistent and Accurate Ab Initio Parametrization of Density Functional Dispersion Correction (DFT-D) for the 94 Elements H-Pu. J. Chem. Phys. 2010, $132,154104$.

(97) Grimme, S.; Ehrlich, S.; Goerigk, L. Effect of the Damping Function in Dispersion Corrected Density Functional Theory. J. Comput. Chem. 2011, 32, 1456-1465.

(98) Weeks, C.; Song, Y.; Suzuki, M.; Chernova, N. A.; Zavalij, P. Y.; Whittingham, M. S. The One Dimensional Chain Structures of Vanadyl Glycolate and Vanadyl Acetate. J. Mater. Chem. 2003, 13, $1420-1423$.

(99) Allen, F. H. The Cambridge Structural Database: A Quarter of a Million Crystal Structures and Rising. Acta Crystallogr., Sect. B: Struct. Sci. 2002, 58, 380-388.

(100) Grimme, S. Semiempirical GGA-Type Density Functional Constructed with a Long-Range Dispersion Correction. J. Comput. Chem. 2006, 27, 1787-1799.

(101) Nanthamathee, C. Thermoresponsive Behaviour of Metal Organic Frameworks. Ph.D. Thesis, University of Manchester, Manchester, U.K., 2013.

(102) Han, S. S.; Goddard, W. A., III Metal-Organic Frameworks Provide Large Negative Thermal Expansion Behavior. J. Phys. Chem. C 2007, 111, 15185-15191.

(103) Greathouse, J. A.; Allendorf, M. D. Force Field Validation for Molecular Dynamics Simulations of IRMOF-1 and Other Isoreticular Zinc Carboxylate Coordination Polymers. J. Phys. Chem. C 2008, 112, 5795-5802.

(104) Tafipolsky, M.; Schmid, R. Systematic First Principles Parameterization of Force Fields for Metal-Organic Frameworks 
Using a Genetic Algorithm Approach. J. Phys. Chem. B 2009, 113,

1341-1315.

(105) Neimark, A. V.; Coudert, F.-X.; Triguero, C.; Boutin, A.; Fuchs, A. H.; Beurroies, I.; Denoyel, R. Structural Transitions in MIL53(Cr): View from the Outside and Inside. Langmuir 2011, 27, 47344741. 